\title{
深海、沙漠、火山、极地来源链霉菌新天然产物(2009～2020)
}

\author{
江 婷 $a$ 蒲 洪 ${ }^{a}$ 段燕文 ${ }^{a, b, c}$ 颜晓晖 $a, d$ 黄 勇*,a,c \\ ( ${ }^{a}$ 中南大学湘雅国际转化医学联合研究院 长沙 410013) \\ ( $b$ 组合生物合成与天然产物药物湖南省工程研究中心 长沙 410011) \\ ( $c$ 新药组合生物合成国家地方联合工程研究中心 长沙 410011) \\ ( ${ }^{d}$ 天津中医药大学中医药研究院 天津 301617)
}

\begin{abstract}
摘要 链需菌是天然产物的重要来源, 但从土壤来源等普通生态环境笁选出的链霉菌中发现新化合物的比例有所降 低. 深海、沙漠、火山或极地属于极端环境, 从中分离出的链霉菌因其特殊气候、地质或营养条件等的差异, 有可能产 生较多新颖天然产物. 综述了 2009 年至 2020 年来从深海、沙漠、火山或极地链霉菌中发现的 155 个新天然产物, 介 绍了它们的结构和生物活性.
\end{abstract}

关键词＼cjkstart链霉菌; 深海; 火山; 沙漠; 天然产物; 生物活性

\section{New Natural Products of Streptomyces Sourced from Deep-Sea, Desert, Volcanic, and Polar Regions from 2009 to 2020}

\author{
Jiang, Ting ${ }^{a} \quad \mathrm{Pu}$, Hong $^{a} \quad$ Duan, Yanwen ${ }^{a, b, c} \quad$ Yan, Xiaohui ${ }^{a, d} \quad$ Huang, Yong ${ }^{*, a, c}$ \\ ( ${ }^{a}$ Xiangya International Academy of Translational Medicine, Central South University, Changsha 410013) \\ $\left({ }^{b}\right.$ Hunan Engineering Research Center of Combinatorial Biosynthesis and Natural Product \\ Drug Discovery, Changsha 410011) \\ ( ${ }^{c}$ National Engineering Research Center of Combinatorial Biosynthesis for Drug Discovery, Changsha 410011) \\ ( ${ }^{d}$ Institute of Traditional Chinese Medicine, Tianjin University of Traditional Chinese Medicine, Tianjin 301617)
}

\begin{abstract}
Streptomyces are important sources of natural products. However, it is more challenging to discover novel compounds from Streptomyces strains isolated from common environmental samples, such as typical soil samples. Due to the unique climates, varying geological and nutrition conditions, deep-sea, desert, volcanic and polar environments are extreme habitats for Streptomyces. These unique habitats are regarded as rare and under-explored niches of new Streptomyces strains and their natural products. The recent advancement of the structures and bioactivities of 155 natural products is reviewed, produced by various Streptomyces strains isolated from these extreme environments from 2009 to 2020.

Keywords Streptomyces; deep-sea; volcano sediments; desert; polar regions; natural products; biological activity
\end{abstract}

链霉菌是生物活性天然产物的重要来源, 包括抗生 素、免疫抑制剂、小分子抗癌药物和其它众多的具有生 物活性的化合物 ${ }^{[1-6]}$. 例如, 在被称为 “抗生素发现黄金 时代” 的 1940 年到 1970 年, 从土壤链霉菌中发现了迄 今仍在使用的大量抗生素, 包括两性霰素 B 等多烯大环 内酯、四环素等芳香聚酮和链霉素等氨基糖苷类化合 物 ${ }^{[5,7]}$. 源于链霉菌的天然产物虽然只占目前已分离的 微生物次级代谢产物的 $39 \%$, 但产生了近 80\%具有生物
活性的化合物 ${ }^{[8]}$. 大量链需菌来源的天然产物已被发现, 导致近年来从土壤来源等普通生态环境篎选出的链霉 菌中发现新化合物的比例有所降低 ${ }^{[7,9]}$. 由于深海、沙 漠、火山或极地属于极端环境, 从中分离出的链霉菌因 其特殊气候、地质或营养条件等差异, 有可能产生较多 新颖天然产物. 因此, 人们近年来从深海、沙漠、火山 沉积物或极地中分离出多株链需菌, 并从中发现了较多 的新天然产物 ${ }^{[10]}$. 例如, Kamjam 等 ${ }^{[11]}$ 对 2006 年到 2016

\footnotetext{
* Corresponding author. E-mail: jonghuang@csu.edu.cn

Received October 5, 2020; revised November 24, 2020; published online December 18, 2020.

Project supported by the National Natural Science Foundation of China (Nos. 81473124, 81872779), and the Fundamental Research Funds for the Central Universities of Central South University (CSU) (No. 2019zzts774).

国家自然科学基金(Nos. 81473124, 81872779)、中南大学中央高校基本科研业务费专项资金(No. 2019zzts774)资助项目.
} 
年发现的源自深海放线菌的天然产物进行了总结, 发现 链䨋菌产生的天然产物最多, 并且大部分具有抗菌或抗 肿瘤活性. 鞠建华和马俊英等 ${ }^{[12]}$ 介绍了海洋链霉菌基 因组挖掘取得的成果和挑战. 朱伟明等[13]综述了 2010 年 1 月到 2013 年 2 月已报道的 895 个海洋微生物新天 然产物, 这些化合物具有高度的化学多样性和生物活 性. 智利北部的阿塔卡马沙漠被认为是地球上最干旱的 极端环境之一, Rateb 等 ${ }^{[14]}$ 总结了 2017 年之前从智利北 部极度干旱的阿塔卡马沙漠分离的放线菌中获得的 46 个天然产物, 并讨论了其结构和生物活性. Sivalingam 等 ${ }^{[15]}$ 综述了 2009 年至 2019 年源至深海、沙漠、低温和 火山沉积物微生物的具有抗菌和细胞毒性的化合物.

本文在 Web of Science 数据库和中国知网分别以链 霉菌(Streptomyces)和深海(deep-sea)、沙漠(desert)、火山 (volcano)、极地、南极、北极(polar regions, Antarctic, Arctic)作为关键词检索相关研究论文. 这些发表在 2009 年到 2020 年 8 月的论文一共报道了 155 个新天然产物. 本文较系统地介绍了这些化合物的结构和生物活性.

\section{1 深海}

\section{1 深海生态系统}

以土壤链霉菌为来源发现新菌株和新天然产物的 颇具挑战性. 海洋生态系统是地球最重要的生态环境: 海洋占地球表面积 $70 \%$, 其中大部分深度超过了 1000 $\mathrm{m}$, 而且全球海洋中有 16 条海沟深度超过 $7000 \mathrm{~m}$. 在整 个海洋生态系统中, 有 $90 \%$ 以上被认为是具有许多不同 特征的深海 ${ }^{[16]}$. 深海微生物的生活环境与浅海微生物 有很大差异, 例如高盐、高压、低营养、低温或局部高 温和无光照等. 生活在深海的链霉菌等微生物为了在无 光、低氧和极高压力的条件下生存, 需要进化出独特的 生理生化过程及代谢方式 ${ }^{[16]}$. 因此, 深海被认为是物种 和生物活性分子发现的最重要的资源之一. 绝大多数已 知的海洋天然产物都源于浅海: 在大约 30000 个已知的 海洋天然产物中, 只有约 $2 \%$ 从深海中分离 ${ }^{[17]}$. 深海来 源的天然产物在生物活性篮选中的阳性率较高, 特别是 其抗增殖活性 ${ }^{[18]}$. 超过 $50 \%$ 的深海天然产物对肿瘤细胞 株有一定的抗增殖活性 ${ }^{[19]}$. 例如, 含有氯原子的海洋环 肽 cryptophycin 的抗肿瘤活性是长春花碱或紫杉醇的 100 至 1000 倍 ${ }^{[20]}$.

近年来, 随着深海勘探技术和微生物分离方法的进 步, 研究人员从海洋环境中分离出多种微生物, 例如 2006 2016 年报道的深海放线菌新物种中有 13 属 21 种, 以皮生球菌属最多, 其次是链霉菌和疮孢菌属 ${ }^{[21]}$. Skropeta $^{[16]}$ 发现深海是生物多样性很丰富的地方: 每平 方英尺的海泥上可发现十多种未知生物, 超过雨林和珊
瑚礁. 深海微生物在极端环境中独特的生存方式, 为从 中发现新天然产物提供了契机 ${ }^{[22]}$.

\section{2 从深海链霉菌中分离的新天然产物}

通过高效液相色谱二极管阵列篮选, Fiedler 等在来 源于加那利盆地的深海链需菌 NTK937 和 NTK935 的提 取物中, 分别检测到新型苯并噁唑 Caboxamycin (1) ${ }^{[23]}$ 和苯并噁嗪 Benzoxacystol (2) ${ }^{[24]}$. 与含有两个苯并噁唑 单元的 UK-1 ${ }^{[25]} 、 A J 19561^{[26]}$ 和 Nataxazole $e^{[27]}$ 相比, 只含 有一个苯并噁唑单元的 1 的细胞毒性更低. 化合物 1 对 枯草芽孢杆菌等革兰氏阳性菌有一定的抗菌活性(表 1). 另外，该化合物对磷酸二酯酶具有抑制作用，而该酶是 治疗哮喘性炎症和慢性阻塞性肺疾病的潜在靶点. 化合 物 2 对糖原合成激酶-3 $\beta$ 有抑制作用, 并对小鼠成纤维 细胞有微弱的抗增殖作用 ${ }^{[24]}$. Losada 等 ${ }^{[28]}$ 通过基因敲除 和异源表达确定了链霉菌 NTK937 中 Caboxamycin 的生 物合成基因族, 并在突变菌株中分离出 3'-Hydroxy-

Caboxamycin (3). 从巴哈马群岛 $1618 \mathrm{M}$ 深的海洋沉积 物中分离的链霉菌 CNR-698, 可以产生 6/6/5 三环吡咯 醌 Ammosamides A (4) 和 B (5). 化合物 4 含有一个独特 的 $\Gamma$ 硫代内酰胺. Ammosamides A (4) 和 B (5)对结肠癌细 胞 HCT-116 有优异的细胞毒性, $\mathrm{IC}_{50}$ 为 $320 \mathrm{nmol} \cdot \mathrm{L}^{-1}[29]$.

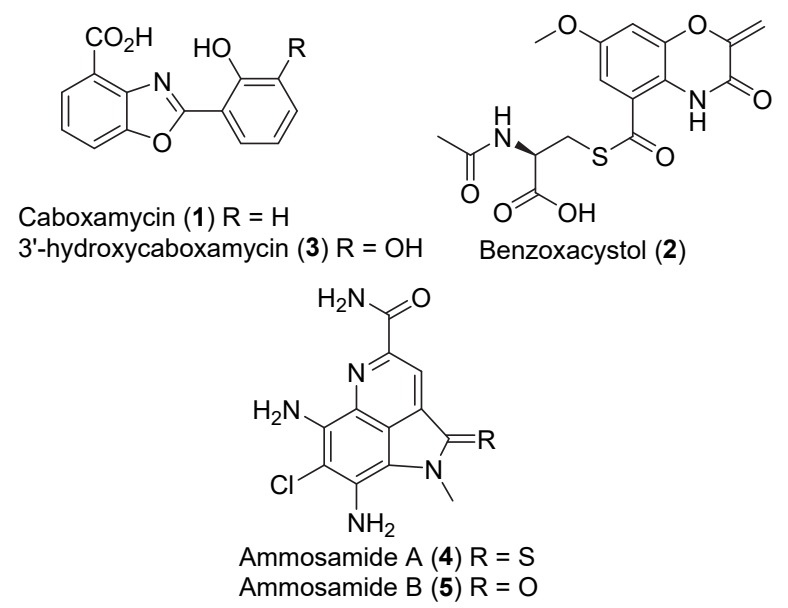

Lobophorins 属于螺环乙酰乙酸内酯类糖苷 ${ }^{[30-32],}$ 其骨架结构与抗生素 Kijanimicin 相似, 具有优异的生物 活性 ${ }^{[33]}$. 鞠建华等 ${ }^{[36]}$ 从中国南海来源的链需菌 SCSIO 01127 中分离 Lobophorins $\mathrm{E} \sim \mathrm{F}(6 \sim 7)$; 张立新等 ${ }^{[32]}$ 发 现了 Lobophorin G (8). 2015 年，胡江春等从中国南海链 霉菌 $12 \mathrm{~A} 35$ 分离到了 Lobophorins $\mathrm{H} \sim \mathrm{I}(\mathbf{9} \sim \mathbf{1 0})^{[34]}$ 和 $\mathrm{J}$ $(\mathbf{1 1})^{[35]} ; 2017$ 年 Blanco 等 ${ }^{[36]}$ 从来自于坎塔布里亚海中部 $1800 \mathrm{M}$ 深处分离的链霉菌 M-207 中, 发现了 Lobophorin K (12). 孙宇辉等 ${ }^{[37]}$ 最近也从深海链霉菌 4506 中分离出 Lobophorins $\mathrm{L} \sim \mathrm{M}(\mathbf{1 3} \sim 14)$. 化合物 $6 、 7$ 对金黄色葡萄球菌 ATCC 29213 具有抗菌活性，其最小 
表 1 深海链需菌的新天然产物 $a$

Table 1 New compounds from deep-sea-derived Streptomyces

\begin{tabular}{|c|c|c|c|c|c|}
\hline Compd. & Source & Bioactivity (MIC or $\mathrm{IC}_{50}$ ) & Depth/m & Region & Year \\
\hline Caboxamycin (1) & NTK 937 & $\begin{array}{l}\text { B. subtilis/S. lentus: } 8 \sim 20 \mu \mathrm{mol} \cdot \mathrm{L}^{-1} \mathrm{AGS} / \\
\text { HepG2/MCF-7: } 28.6 \sim 29.4 \mu \mathrm{mol} \cdot \mathrm{L}^{-1}\end{array}$ & 3814 & Canary Basin & $2009^{[23]}$ \\
\hline Benzoxacystol (2) & NTK 935 & $\begin{array}{l}\text { Inhibitory activity against glycogen synthase } \\
\text { kinase- } 3 \beta\end{array}$ & 3814 & Canary Basin & $2011^{[24]}$ \\
\hline 3'-hydroxycaboxamycin (3) & NTK 937 & - & 3814 & Canary Basin & $2017^{[28]}$ \\
\hline Ammosamides A/B (4/5) & CNR-698 & HCT-116: $320 \mathrm{nmol} \cdot \mathrm{L}^{-1}$ & 1618 & Bahamas & $2009^{[29]}$ \\
\hline Lobophorins E/F (6/7) & SCSIO 01127 & $\begin{array}{l}\text { S. aureus/E. faecalis: } 8 \mu \mathrm{g} / \mathrm{mL}(6) \mathrm{SF}-268 / \\
\mathrm{MCF}-7 / \mathrm{H} 460: 2.93 \sim 6.82 \mu \mathrm{mol} \cdot \mathrm{L}^{-1} \text { (7) }\end{array}$ & 135 & South China Sea & $2011^{[76]}$ \\
\hline Lobophorins G (8) & MS100061 & $\begin{array}{l}\text { BCG: } 1.56 \mu \mathrm{g} / \mathrm{mL} \text { B. subtilis: } 3.125 \mu \mathrm{g} / \mathrm{mL} \\
\text { M. tuberculosis H37Rv: } 16 \mu \mathrm{g} / \mathrm{mL}\end{array}$ & NA & South China Sea & $2013^{[32]}$ \\
\hline Lobophorins H/I (9/10) & $12 \mathrm{~A} 35$ & $\begin{array}{l}\text { B. subtilis CMCC 63501: } 1.57 \mu \mathrm{g} / \mathrm{mL} \\
\text { (9) } 50 \mu \mathrm{g} / \mathrm{mL} \text { (10) }\end{array}$ & 2134 & South China Sea & $2013^{[34]}$ \\
\hline Lobophorins J (11) & $12 \mathrm{~A} 35$ & - & 2134 & South China Sea & $2015^{[35]}$ \\
\hline Lobophorin K (12) & M-207 & $\begin{array}{l}\text { S. aureus: } 40 \sim 80 \mu \mathrm{g} / \mathrm{mL} \mathrm{MiaPaca-} 2 / \\
\text { MCF-7/THLE-2: } 6.3 \sim 34.0 \mu \mathrm{mol} \cdot \mathrm{L}^{-1}\end{array}$ & 180 & Cantabrian Sea & $2017^{[36]}$ \\
\hline Lobophorin L/M (13/14) & 4506 & $\begin{array}{l}\text { M. luteus/B. huringiensis: } 4 \sim 8 \mu \mathrm{g} / \mathrm{mL} \\
\text { (13) }\end{array}$ & NA & South China Sea & $2020^{[37]}$ \\
\hline Spiroindimicins $A \sim D(\mathbf{1 5} \sim \mathbf{1 8})$ & SCSIO 03032 & $\begin{array}{l}\text { CCRF-CEM/B16/H460: 4 12 } \mu \mathrm{g} / \mathrm{mL} \\
\text { (except 15) }\end{array}$ & 3412 & Indian Ocean & $2012^{[44]}$ \\
\hline Spiroindimicins G/H (19/20) & SCSIO 03032 & $\begin{array}{l}\text { SF-268/MCF-7/HepG2/A549: 10.28 } \\
33.02 \mu \mathrm{mol} \cdot \mathrm{L}^{-1}\end{array}$ & 3412 & South China Sea & $2019^{[45]}$ \\
\hline Indinicins $A \sim \mathrm{E}(\mathbf{2 1} \sim \mathbf{2 5})$ & SCSIO 03032 & $\begin{array}{l}\text { SF-268/MCF-7/H460/HepG2: } 9.7 \sim \\
44.6 \mu \mathrm{mol} \cdot \mathrm{L}^{-1}\end{array}$ & 3412 & Indian Ocean & $2014^{[46]}$ \\
\hline Indinicins F/G (26/27) & SCSIO 03032 & - & 3412 & Indian Ocean & $2019^{[45]}$ \\
\hline Lynamicins F/G (28/29) & SCSIO 03032 & $\begin{array}{l}\mathrm{SF}-268 / \mathrm{H} 460 / \mathrm{HepG} 2 / \mathrm{MCF}-7:>100 \\
\mu \mathrm{mol} \cdot \mathrm{L}^{-1}\end{array}$ & 3412 & Indian Ocean & $2014^{[46]}$ \\
\hline Heronamides $\mathrm{D} \sim \mathrm{F}(\mathbf{3 0} \sim \mathbf{3 2})$ & SCSIO 03032 & - & 3412 & Indian Ocean & $2014^{[49]}$ \\
\hline Dionemycin (33)/34 & SCSIO 11791 & $\begin{array}{l}\text { S. aureus ATCC 29213/MRSA:0.5 2 } \\
\mu \mathrm{g} / \mathrm{mL} \text { H460/MDA-MB-231/HCT-116/ } \\
\text { HepG2/MCF10A: } 3.1 \sim 11.2 \mu \mathrm{mol} \cdot \mathrm{L}^{-1}\end{array}$ & 1765 & South China Sea & $2020^{[22]}$ \\
\hline Grincamycins $B \sim F(35 \sim 39)$ & SCSIO LR32 & $\begin{array}{l}\text { HepG2/SW-1990/HeLa/H460/MCF-7/ } \\
\text { B16: 1.1 } 231 \mu \mathrm{mol} \cdot \mathrm{L}^{-1}(\text { except 39) }\end{array}$ & 3370 & South China Sea & $2012^{[50]}$ \\
\hline Grincamycins G/H (40/41) & SCSIO LR32 & Jurkat T-cell: $3.0 \mu \mathrm{mol} \cdot \mathrm{L}^{-1}$ (41) & 3370 & South China Sea & $2012^{[51]}$ \\
\hline Grincamycins $I \sim K(\mathbf{4 2} \sim \mathbf{4 4})$ & SCSIO LR32 & $\begin{array}{l}\text { MDA-MB-435/MDA-MB-231/H460/ } \\
\text { HCT-116/HepG2/MCF10A: } 2.43 \sim \\
25.87 \mu \mathrm{mol} \bullet \mathrm{L}^{-1}\end{array}$ & 3370 & South China Sea & $2018^{[52]}$ \\
\hline Marfuraquinocins $A \sim D(\mathbf{4 5} \sim \mathbf{4 8})$ & SCSIO 3406 & $\begin{array}{l}\text { S. aureus ATCC } 29213 / \mathrm{MRSE}: 8 \sim 64 \\
\mu \mathrm{g} / \mathrm{mL} \text { SF } 268 / \mathrm{MCF}-7 / \mathrm{H} 460 / \mathrm{HepG} 2: \\
3.6 \sim 26.9 \mu \mathrm{mol} \cdot \mathrm{L}^{-1}\end{array}$ & 3536 & South China Sea & $2013^{[54]}$ \\
\hline Phenaziterpenes $\mathrm{A} \sim \mathrm{B}(\mathbf{4 9 / 5 0})$ & SCSIO 3406 & $\begin{array}{l}\text { A. hydrophila ATCC 7966: } 64 \mu \mathrm{g} / \mathrm{mL} \\
\text { (49) SF268/MCF-7/HepG2: } 9.3 \sim 54.5 \\
\mu \mathrm{mol} \cdot \mathrm{L}^{-1}\end{array}$ & 3536 & South China Sea & $2013^{[54]}$ \\
\hline Sungsanpin (51) & SNJ013 & Inhibitory activity against A549 & 138 & Jeju Island & $2013^{[55]}$ \\
\hline Strepsesquitriol (52) & SCSIO 10355 & $\begin{array}{l}\text { Inhibitory activity against lipopoly } \\
\text { saccharide induced } T N F \alpha \text { production }\end{array}$ & 3412 & Indian Ocean & $2013^{[56]}$ \\
\hline
\end{tabular}




\begin{tabular}{|c|c|c|c|c|c|}
\hline Compd. & Source & Bioactivity (MIC or $\mathrm{IC}_{50}$ ) & Depth/m & Region & Year \\
\hline Tetroazolemycins A/B (53/54) & FXJ8.012 & K. pneumoniae/Metal ion-binding activity & NA & Indian Ocean & $2013^{[57]}$ \\
\hline Mycemycins $\mathrm{C} \sim \mathrm{E}(\mathbf{5 5} \sim \mathbf{5 7})$ & FXJ8.012 & Anti-HIV-1 reverse transcriptase activity & NA & Indian Ocean & $2015^{[67]}$ \\
\hline Champacyclin (58) & $\mathrm{C} 42$ & Erwinia amylovora: $25 \mu \mathrm{mol} \cdot \mathrm{L}^{-1}$ & 241 & Baltic Sea & $2013^{[59]}$ \\
\hline Xiamenmycins C/D (59/60) & M1-94P & WI26: $15 \mu \mathrm{g} / \mathrm{mL} / 30 \mu \mathrm{g} / \mathrm{mL}$ & 2628 & Pacific & $2013^{[60]}$ \\
\hline Desotamides $\mathrm{B} \sim \mathrm{D}(\mathbf{6 1} \sim \mathbf{6 3})$ & SCSIO ZJ46 & $\begin{array}{l}\text { S. pnuemoniae NCTC 7466/S. aureus ATCC } \\
\text { 29213/MRSE Shhs-E1: } 12.5 \sim 32.0 \mu \mathrm{g} / \mathrm{mL}\end{array}$ & 3536 & South China Sea & $2014^{[64]}$ \\
\hline Bafilomycins B1/C1 (64/65) & NA4 & $\begin{array}{l}\text { Inhibitory activities against Fusarium } \\
\text { spp. and } R \text {. solani }\end{array}$ & 1464 & South China Sea & $2015^{[65]}$ \\
\hline Marformycins $A \sim F(66 \sim 71)$ & SCSIO 10141 & M. luteus: $0.06 \sim 4.0 \mu \mathrm{g} / \mathrm{mL}$ & 1396 & South China Sea & $2014^{[66]}$ \\
\hline Marangucyclines A/B (72/73) & SCSIO 11594 & $\begin{array}{l}\text { E. faecalis ATCC29212: } 64.0 \mu \mathrm{g} / \mathrm{mL} \\
\text { A594/CNE2/HepG2/MCF-7: } 0.24 \sim \\
0.56 \mu \mathrm{mol} \cdot \mathrm{L}^{-1}(\mathbf{7 3})\end{array}$ & 2403 & South China Sea & $2015^{[67]}$ \\
\hline $74 / 75$ & PKU-MA01297 & - & 3202 & Indian Ocean & $2019^{[68]}$ \\
\hline 76 & SCSIO 04496 & - & 3536 & South China Sea & $2016^{[77]}$ \\
\hline Tunicamycin E (77) & SCSIO S15077 & $\begin{array}{l}\text { B. thuringiensis/C. albicans CMCC (F) } \\
98001 / \text { C. albicans ATCC 96901: 0.5 } \\
32 \mu \mathrm{g} / \mathrm{mL}\end{array}$ & 3536 & South China Sea & $2018^{[69]}$ \\
\hline Atratumycin (78) & SCSIO ZH16 & $\begin{array}{l}\text { M. tuberculosis } \mathrm{H} 37 \mathrm{Ra} / \mathrm{H} 37 \mathrm{Rv}: 3.8 / \\
14.6 \mu \mathrm{mol} \cdot \mathrm{L}^{-1}\end{array}$ & 3536 & South China Sea & $2019^{[61]}$ \\
\hline $79 / 80$ & OUCMBZ-4112 & - & 2206 & South China Sea & $2016^{[62]}$ \\
\hline 81 & OUCMDZ-2167 & - & 2061 & South China Sea & $2016^{[63]}$ \\
\hline Butenolids 1/2 (82/83) & TP-A0873 & $\begin{array}{l}\text { Peroxisome proliferator activated receptor- } \\
\text { PPAR } \alpha \text { agonists }\end{array}$ & NA & Toyama Bay & $2014^{[78]}$ \\
\hline Ahpatinins Ac/Pr (84/85) & ACT232 & $\begin{array}{l}\text { Aspartic protease inhibitors: } 0.01 \sim 0.05 \\
\mu \mathrm{mol} \cdot \mathrm{L}^{-1}\end{array}$ & 1174 & Sagami Bay & $2014^{[64]}$ \\
\hline Fradiamines A/B (86/87) & MM456M-F7 & Clostridium difficile: $8 \sim 32 \mu \mathrm{g} / \mathrm{mL}$ & 806 & Sagami Bay & $2017^{[71]}$ \\
\hline $88 \sim 90$ & M-157 & HepG2: $51.5 \mu \mathrm{mol} \cdot \mathrm{L}^{-1}$ & 2000 & Cantabrian Sea & $2018^{[72]}$ \\
\hline Anthracimycin B (91) & M-169 & $\begin{array}{l}\text { S. aureus MB5393/S. aureus ATCC29213/ } \\
\text { E. faecium CL144754/E. faecalis CL144492 } \\
0.125 \sim 8.0 \mu \mathrm{mol} \cdot \mathrm{L}^{-1}\end{array}$ & 1500 & Cantabrian Sea & $2018^{[74]}$ \\
\hline Flaviogeranins $\mathrm{B} \sim \mathrm{D}(\mathbf{9 2} \sim \mathbf{9 4})$ & B9173 & $\begin{array}{l}\text { S. aureus/M. smegmatis: } 5.0 \sim 35.7 \mu \mathrm{g} / \mathrm{mL} \\
\text { A549/Hela: } 0.4 \sim 50.2 \mu \mathrm{mol} \cdot \mathrm{L}^{-1}\end{array}$ & NA & Pacific Ocean & $2020^{[75]}$ \\
\hline
\end{tabular}

${ }^{a}$ NA: Not specified.

抑菌浓度(MIC) 分别为 6.25 和 $32 \mu \mathrm{g} / \mathrm{mL}$. 与早期报道的 Lobophorin B 相比, 6 和 7 中 C-32 羟基的缺失增强了它 们对金黄色葡萄球菌 ATCC 29213 的抗菌活性. 化合物 8 表现出较强的抗卡介苗活性, 其 MIC 为 $1.56 \mu \mathrm{g} / \mathrm{mL}$. 化合物 9 对枯草芽孢杆菌 CMCC63501 抑制作用较强, 具有与氨芳青霉素相当的抗菌活性. 化合物 13 对藤黄 微球菌和苏云金芽孢杆菌的 MIC 分别为 4 和 $8 \mu \mathrm{g} / \mathrm{mL}$, 而 14 在浓度为 $128 \mu \mathrm{g} / \mathrm{mL}$ 时对这两种细菌均无抑制作 用. 因此, 化合物 $6 \sim 14$ 的体外抗菌效果可能与螺环乙 酰乙酸内酯骨架上糖基取代的数量和其后修饰相关. 另 外, 7 对神经癌细胞 SF-268、乳腺癌细胞 MCF-7 和人大
细胞肺癌细胞 $\mathrm{H} 460$ 有良好的细胞毒活性, $\mathrm{IC}_{50}$ 分别为 6.82、 2.93 和 $3.16 \mu \mathrm{mol} / \mathrm{L}$. 化合物 $\mathbf{1 2}$ 对 MCF-7、人胰 腺癌细胞 MiaPaca-2 和人肝细胞 THLE-2 有细胞毒性, $\mathrm{IC}_{50}$ 分别为(23.0 \pm 8.9$) 、(34.0 \pm 85.1)$ 和 $(6.3 \pm 8.2) \mu \mathrm{mol} / \mathrm{L}$.

色氨酸二聚体是一类独特的生物碱, 其结构多样, 生物活性优异 ${ }^{[38-40]}$. 目前有多种色氨酸二聚体已经获批 准用于癌症的临床使用或正在临床试验 ${ }^{[41-42]}$. 例如, 以 吲哚咔唑骨架为特征的化合物 Midostaurin 已获批准用 于治疗具有 FLT3-ITD 突变的急性髓系白血病 ${ }^{[43]}$. 张长 生等从印度洋 $3412 \mathrm{~m}$ 深处采集的深海沉积物中, 分离 出链霉菌 SCSIO 03032. 该菌株及其突变菌株产生了一 


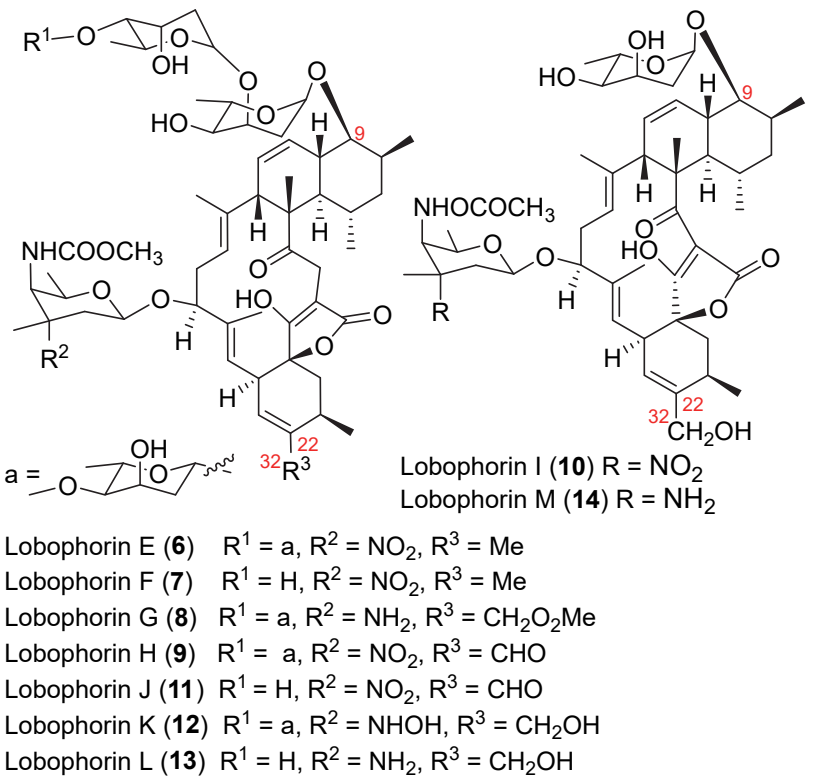

系列结构独特的色氨酸二聚体 Spiroindimicins $\mathrm{A} \sim \mathrm{D}$ $(\mathbf{1 5} \sim \mathbf{1 8})^{[44]}$ 和 $\mathrm{G} \sim \mathrm{H}(\mathbf{1 9} \sim \mathbf{2 0})^{[45]} 、$ Indimicins $\mathrm{A} \sim \mathrm{G}(\mathbf{2 1} \sim$ 27) ${ }^{[45-46]}$ 以及 Lynamicins $F \sim G(\mathbf{2 8} \sim 29)^{[46]}$. 活性评价发 现 16 对急性淋巴白血病细胞 CCRF-CEM、小鼠黑色素 瘤细胞 B16 和 $\mathrm{H} 460$ 等具有良好的细胞毒性, 其 $\mathrm{IC}_{50}$ 分 别为 $4 、 5$ 和 $12 \mu \mathrm{g} / \mathrm{mL}^{[47]}$. 化合物 $\mathbf{1 7}$ 和 $\mathbf{1 8}$ 对 HepG2 和 H460 有中等抑制活性, 而 $19 \sim 20$ 对四种癌细胞系 SF-268、MCF-7、肝癌细胞 HepG2 和非小细胞肺癌细胞 A549 具有一定的细胞毒性 ${ }^{[48]}$. 双吲哚生物碱 22 对 MCF-7 的 $\mathrm{IC}_{50}$ 值大于 $10.0 \mu \mathrm{mol} \cdot \mathrm{L}^{-1}$, 而对 $\mathrm{H} 460 、 \mathrm{HepG} 2$ 和 SF-268 无细胞毒性. 结构独特的大环聚酮内酰胺 Heronamides $\mathrm{D} \sim \mathrm{F}(\mathbf{3 0} \sim \mathbf{3 2})$ 也由张长生等从链霰菌 SCSIO 03032 分离获得, 但它们未显示出明显抗菌活性 或细胞毒性 ${ }^{[49]}$. 鞠建华等从深海链霉菌 SCSIO 11791 中 发现了氯代双吲哚生物碱 Dionemycin (33)及其类似物 34. 化合物 33 对 6 株耐甲氧西林金黄色葡萄球菌均有优 异的抗菌活性，其 MIC 为 $1 \sim 2 \mu \mathrm{g} / \mathrm{mL}$. 此外，化合物 33 对人癌细胞株 H460、MDA-MB-231、HCT-116、HepG2 和非癌性 MCF10A 具有细胞毒活性, $\mathrm{IC}_{50}$ 分别为 3.1 $11.2 \mu \mathrm{mol} \cdot \mathrm{L}^{-1}$. 通过构效关系分析发现 C-6"处的氯原子 可能是 33 具有生物活性的关键, 为双吲哚生物碱类化 合物的结构优化提供了线索 ${ }^{[22]}$.

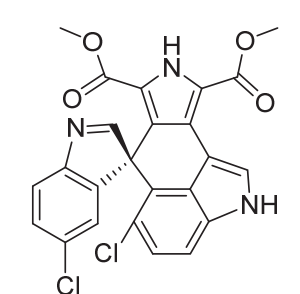

Spiroindimicin A (15)

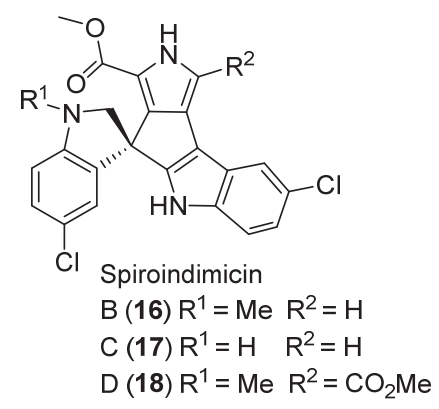<smiles>CNc1ccccc1[C@@H]1c2[nH]c3ccccc3c2-c2c(C(=O)OC)[nH]c(C(=O)OC)c21</smiles>

Spiroindimicin G (19)<smiles>[R]c1c2c(cn1[R])[C@@]1(C)c3cc(Cl)ccc3N(C)[C@H]1c1[nH]c3ccc(Cl)cc3c1-2</smiles>

Indimicin $\mathrm{A}(\mathbf{2 1}) \mathrm{R}^{1}=\mathrm{Me} \mathrm{R}^{2}=\mathrm{H}$ Indimicin $B(22) R^{1}=R^{2}=M e$ Indimicin $\mathrm{C}(\mathbf{2 3}) \mathrm{R}^{1}=\mathrm{H}, \mathrm{R}^{2}=\mathrm{Me}$ Indimicin $D(24) R^{1}=R^{2}=H$<smiles></smiles>

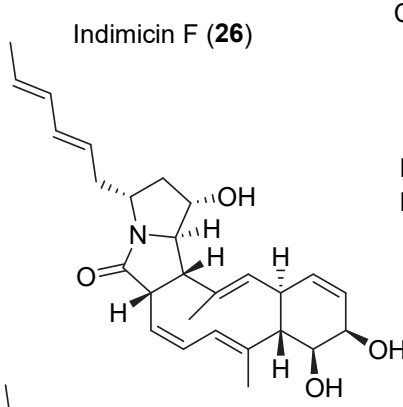

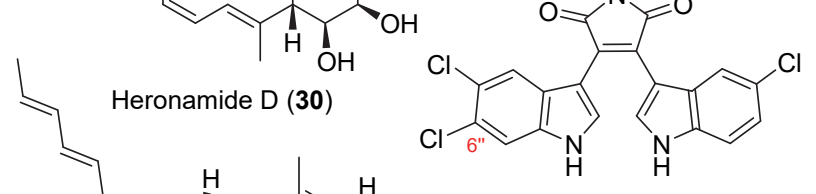

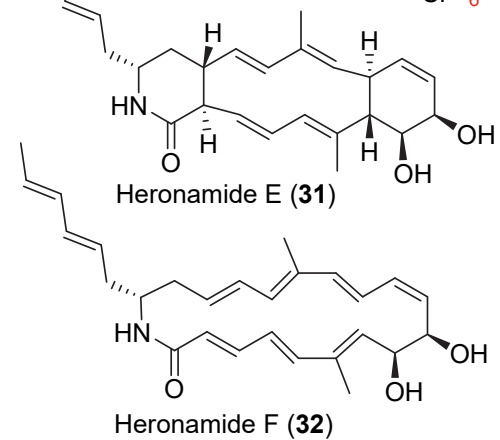
Dionemycin (33)<smiles>COC(=O)c1[nH]c(C(=O)OC)c(-c2c[nH]c3c(Cl)cccc23)c1-c1c[nH]c2c(Cl)cccc12</smiles>
34

鞠建华等从来自中国南海的链霉菌 SCSIO LR32 中 发现了角环素类糖苷 Grincamycins $\mathrm{B} \sim \mathrm{F}(\mathbf{3 5} \sim \mathbf{3 9})^{[50]}$ 、 $\mathrm{G} \sim \mathrm{H}(\mathbf{4 0} \sim \mathbf{4 1})^{[51]} 、 \mathrm{I} \sim \mathrm{K}(\mathbf{4 2} \sim \mathbf{4 4})^{[52]}$ 和 已知的 Grincamycin A. 除 39 外, 这类化合物均对多种人类癌 细胞, 如 HepG2、胰腺癌细胞 SW-1990、MCF-7 和 B16 有较强的细胞毒性, $\mathrm{IC}_{50}$ 为 $1.1 \sim 31 \mu \mathrm{mol} \cdot \mathrm{L}^{-1}$. 最近, 化 合物 35 被发现对两种多形性成胶质细胞瘤细胞 U251 和 091214 的生长和侵袭具有抑制作用，并可以靶向胶 
质瘤干细胞. 因此, 该化合物是一种很有前途的靶向 RHOA 和 PI3K/AKT 的胶质瘤干细胞抑制剂 ${ }^{[53]}$. 与其它 Grincamycins 相比, 39 含有一个独特的六元内酯环和一 个苯酚, 这一扩张的苷元结构可能导致其失去对肿瘤细 胞的毒性. Marfuraquinocins $A \sim D(45 \sim 48)$ 和哌嗪类化 合物 Phenaziterpenes $\mathrm{A} \sim \mathrm{B}(\mathbf{4 9} \sim \mathbf{5 0})$ 产自中国南海的深 海链霉菌 SCSIO 3406 ${ }^{[54]}$. 菜醌倍半萜 45 和 47 对 H460 有细胞毒性, $\mathrm{IC}_{50}$ 分别为 3.7 和 $4.4 \mu \mathrm{mol} / \mathrm{L}$. 化合物 45 、 47 和 48 对金黄色葡萄球菌 ATCC 29213 的 MIC 值为 8.0 $\mu \mathrm{g} / \mathrm{mL}$, 其中 47 和 48 对耐甲氧西林表皮葡萄球菌 shhs$\mathrm{E} 1$ 的 $\mathrm{MIC}$ 为 $8.0 \mu \mathrm{g} / \mathrm{mL}$. Oh 等 ${ }^{[55]}$ 从韩国济州岛 $138 \mathrm{~m}$ 深 处的沉积物中分离出链霉菌 SNJ013, 发现一个含有 15
个氨基酸的套索肽 Sungsanpin (51)，该化合物在对 A549 的侵袭试验中表现出一定的抑制活性.

刘永宏等从产自印度洋的深海链霉菌 SCSIO 10355 发现了具有 5/6/5 三环骨架的倍半萜 Strepsesquitriol (52), 并发现 52 在脂多糖诱导的巨噬细胞的抗炎模型 中，可以抑制 $\mathrm{TNF} \alpha$ 的产生 ${ }^{[56]}$. 黄英等从产自印度洋的 深海链霉菌 FXJ8.012 中发现结构对称的噻唑/噁唑类铁 载体 Tetroazolemycins $\mathrm{A} \sim \mathrm{B}(\mathbf{5 3} \sim \mathbf{5 4})$. 化合物 $\mathbf{5 3} \sim \mathbf{5 4}$ 可 以络合多种金属离子，其锌离子配合物对肺炎克雷伯菌 有一定的抑制作用 ${ }^{[57]}$. 含有 Oxazepinone 稠杂环的 Mycemycins $\mathrm{C} \sim \mathrm{E}(\mathbf{5 5} \sim \mathbf{5 7})$ 也在 $g h t R$ 基因缺失的 FXJ8. $012 \Delta 1741$ 被发现 ${ }^{[58]}$.<smiles>[R]c1ccc2c(c1O)C(=O)c1ccc(CC(C)(O)CC(=O)O)c(O)c1C2=O</smiles>

Grincamycin B (35) $R^{1}=a, R^{2}=b$ Grincamycin $C(36) R^{1}=a, R^{2}=H$ Grincamycin D (37) $R^{1}=c, R^{2}=b$<smiles>[R20]C(O)C1CCCC(c2ccc3c(c2O)C(=O)c2cc4c(O)cc(C)cc4c(O)c2C3=O)C1CC</smiles>
Grincamycin $\mathrm{H}$ (41)<smiles>[R]c1ccc2c(c1O)C(=O)c1cc3c(c(O)c1C2=O)CC(C)(C)CC3=O</smiles>
Grincamycin E (38) $R^{1}=a, R^{2}=b$

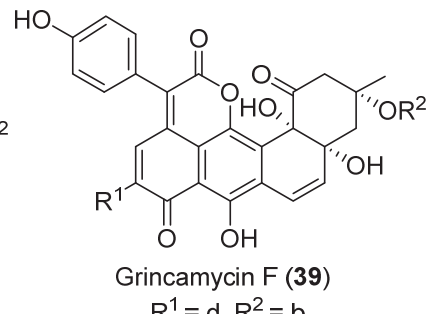
$R^{1}=d, R^{2}=b$

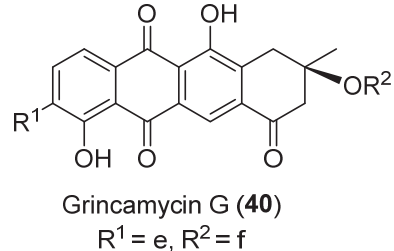
$R^{1}=e, R^{2}=f$<smiles>[R]c1ccc2c(c1O)C(=O)c1ccc3c(c1C2=O)C(=O)CC([R])(C)C3</smiles>
Grincamycin I (42) $R^{1}=a, R^{2}=b$ Grincamycin J (43) $\mathrm{R}^{1}=\mathrm{a}, \mathrm{R}^{2}=\mathrm{H}$

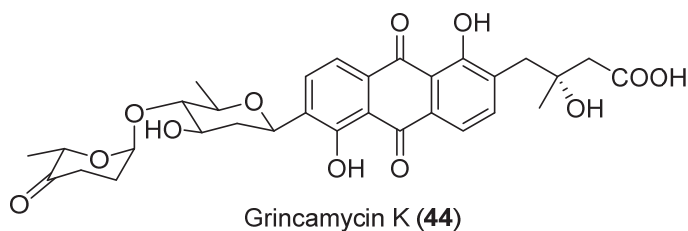<smiles>[R]CC1Oc2c(c(O)cc3c2C(C)(CCC2C(=C)CCCC2(C)C)C3)C(=O)C=C1OC</smiles><smiles>[R]C[C@H]1Oc2c(c(O)cc3c2C(CCC2C(=C)CCCC2(C)C)C3(C)C)C(=O)C=C1OC</smiles>

Marfuraquinocin $\mathrm{B}(47) \mathrm{R}=\mathrm{H}$ Marfuraquinocin $\mathrm{C}(\mathbf{4 6}) \mathrm{R}=\mathrm{OH} \quad$ Marfuraquinocin $\mathrm{D}(\mathbf{4 8}) \mathrm{R}=\mathrm{OH}$

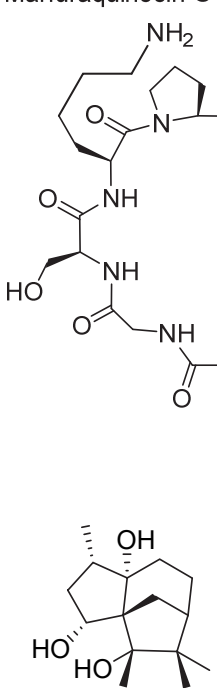

Strepsesquitriol (52)

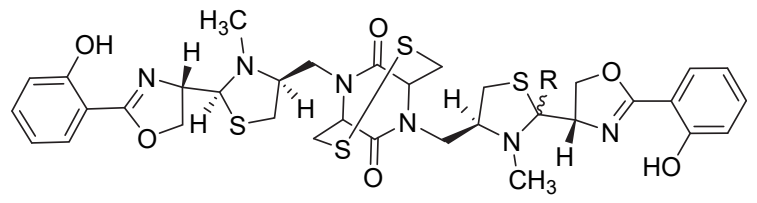

Tetroazolemycin A (53) $\mathrm{R}=\cdots \cdot \mathrm{H}$ Tetroazolemycin B (54) $\mathrm{R}=-\mathrm{H}$<smiles>[R2]c1cccc2nc3c(O/C=C/C(C)CCC=C(C)C)cccc3nc12</smiles>

Phenaziterpene $\mathrm{A}(\mathbf{4 9}) \mathrm{R}=\mathrm{H}$ Phenaziterpene $\mathrm{B}(\mathbf{5 0}) \mathrm{R}=\mathrm{Me}$

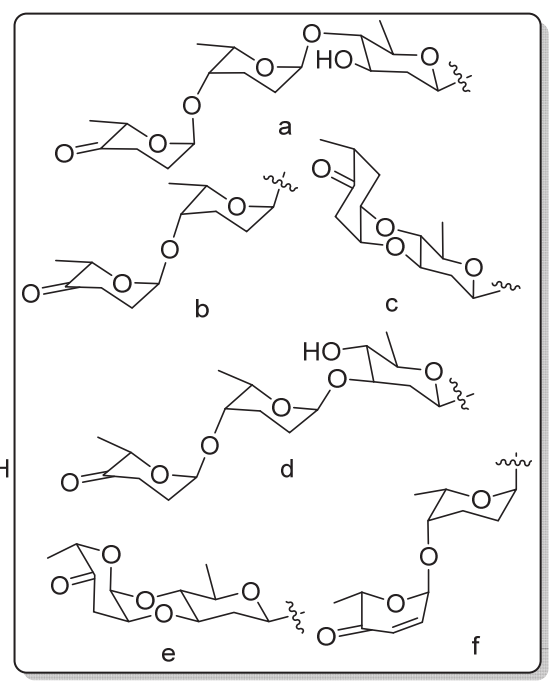

Sungsanpin (51)

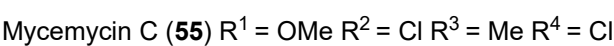
Mycemycin D (56) $R^{1}=\mathrm{NH}_{2} \mathrm{R}^{2}=\mathrm{Cl} \mathrm{R}^{3}=\mathrm{Me} \mathrm{R}^{4}=\mathrm{H}$ Mycemycin E (57) $\mathrm{R}^{1}=\mathrm{NH}_{2} \mathrm{R}^{2}=\mathrm{Cl} \mathrm{R}^{3}=\mathrm{Me} \mathrm{R}^{4}=\mathrm{Cl}$ 
从波罗的海分离的链霉菌 C42 中发现的一个新的 环状八肽 Champacyclin (58), 对梨火疫病病原菌具有一 定的抗菌活性 ${ }^{[59]}$. 徐俊等基于东太平洋深海沉积物的 菌株 M1-94P, 篎选并构建了耐受利福霉素和链霉素的 抗性菌株, 从中分离到苯并吡喃 Xiamenmycins $\mathrm{C} \sim \mathrm{D}$ $(59 \sim 60)$. 活性评价发现化合物 59 60 均能抑制 WI26 (人肺成纤维细胞)的增殖, 将 WI26 分别暴露在浓度为 $15 、 30 \mu \mathrm{g} / \mathrm{mL}$ 的 59、60 中，化合物 59 对 WI26 的抑制 率从第 1 天的 $13.8 \%$ 增加到第 6 天的 $38 \%$; 化合物 $\mathbf{6 0}$ 对 WI26 的抑制率从第 1 天的 $12.8 \%$ 增加到了第 6 天的 $38 \%{ }^{[60]}$. Xiamenmycins 中吡喃环的生物合成由一个黄素

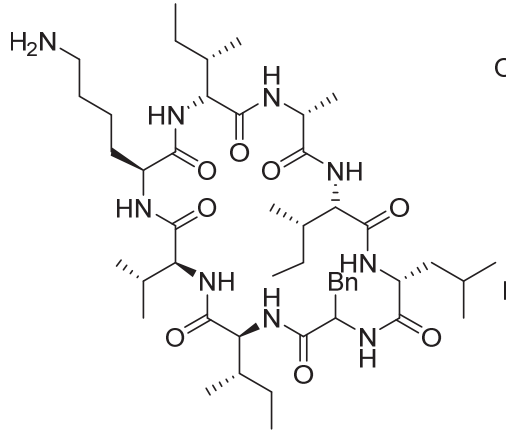

Champcylin (58)<smiles>CC(C)=CCC[C@]1(C)Oc2ccc(C(N)=O)cc2C[C@H]1O</smiles>

Xiangmenmycin $\mathrm{C}(59)$<smiles>COC(=O)[C@H](NC(=O)c1ccc2c(c1)C[C@H](O)[C@](C)(CCC=C(C)C)O2)[C@@H](C)O</smiles>

Xiangmenmycin $\mathrm{D}(\mathbf{6 0})$

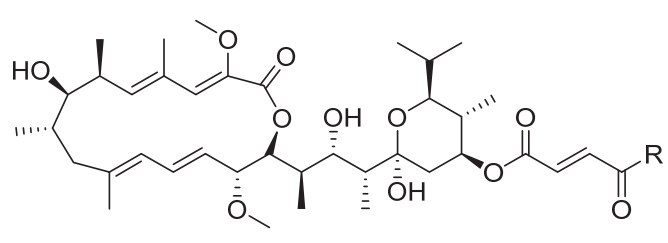

Bafilomycin $\mathrm{C} 1(64) \mathrm{R}=\mathrm{OH}$<smiles>[R]#CNC1=C(O)CCC1=O</smiles>

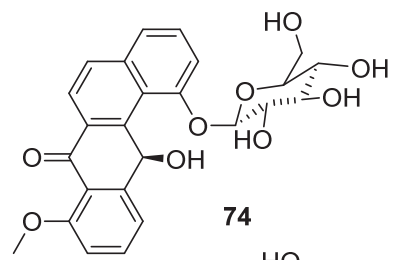

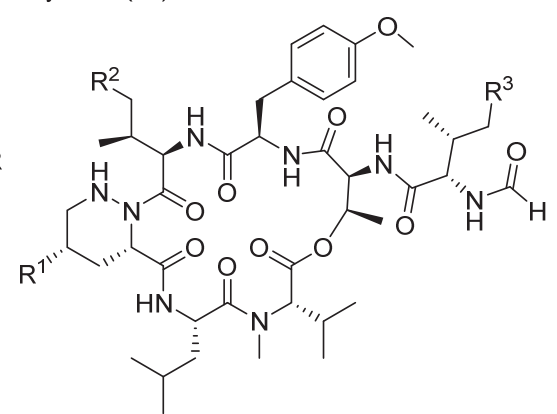

Marformycin $A(66) R^{1}=H ; R^{2}=M e ; R^{3}=H$ Marformycin B (67) $\mathrm{R}^{1}=\mathrm{H} ; \mathrm{R}^{2}=\mathrm{Me} ; \mathrm{R}^{3}=\mathrm{Me}$ Marformycin $\mathrm{C}(68) \mathrm{R}^{1}=\mathrm{OH} ; \mathrm{R}^{2}=\mathrm{H} ; \mathrm{R}^{3}=\mathrm{Me}$ Marformycin $D(69) \mathrm{R}^{1}=\mathrm{OH} ; \mathrm{R}^{2}=\mathrm{Me} ; \mathrm{R}^{3}=\mathrm{Me}$ Marformycin $\mathrm{E}(\mathbf{7 0}) \mathrm{R}^{1}=\mathrm{OH} ; \mathrm{R}^{2}=\mathrm{H} ; \mathrm{R}^{3}=\mathrm{H}$ Marformycin $F(71) \mathrm{R}^{1}=\mathrm{H} ; \mathrm{R}^{2}=\mathrm{H} ; \mathrm{R}^{3}=\mathrm{H}$

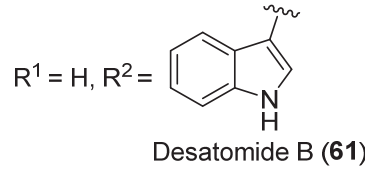<smiles>[R]=CNc1ccc([R]#N)cc1C(=O)O</smiles>

Desatomide C (62)<smiles>[R]=[R]=C1C=CC(N)=C(C(C)(C)C)C1</smiles>

Desatomide D (63)<smiles>[R]c1ccc2c(c1O)C(=O)c1ccc3cc(C)cc(O)c3c1C2=O</smiles>

Marangucycline A (72) $\mathrm{R}=$ $\mathrm{HO} \mathrm{NO}^{-1}$<smiles>CCC(O)C1CCOCC1O</smiles>

Marangucycline $\mathrm{B}(73) \mathrm{R}=$ $0 \Rightarrow$ TOOT<smiles>C/C=C\C=C/c1cccc2c1C(=O)c1cccc(OC)c1C2=O</smiles>

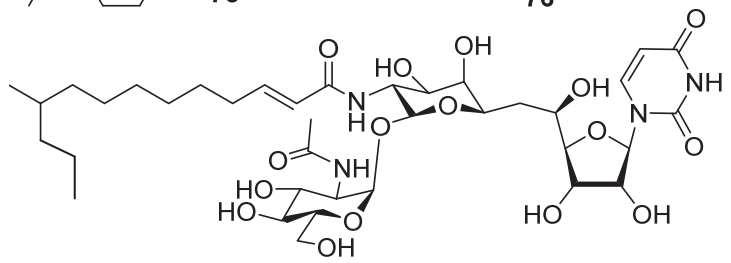

Tunicamycin E (77)

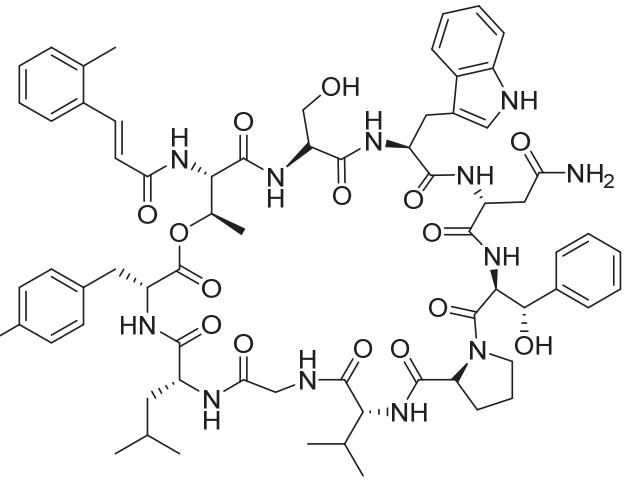

Atratumycin (78)<smiles>CO[C@@H](CC(=O)c1ccc[nH]1)C(N)=O</smiles>

79: 2S; 80: 2R

81<smiles>COC(=O)c1cc(CO)co1</smiles> 
环己肽 61 对金黄色萄球菌 ATCC 29213、肺炎链球 菌 NCTC7466 和金黄色葡萄球菌 Shhs-E1 的 MIC 分别 为 $16.0 、 12.5$ 和 $32.0 \mu \mathrm{g} / \mathrm{mL}$. 化合物 61 和 63 均含有色 氨酸, 这与其较好的抗菌活性相关 ${ }^{[64]}$. 化合物 $64 \sim 65$ 可能用于植物病原真菌的防治 ${ }^{665}$. Marformycins $\mathrm{A} \sim \mathrm{F}$ (66 71) 具有一个独特的 $N$-甲酰基和 5 个非天然氨基酸 残基, 其中 66 70 对藤黄微球菌具有优异的抗菌活性, 其 MIC 分别为 $0.25 、 4.0 、 0.25 、 0.063$ 和 $4.0 \mu \mathrm{g} / \mathrm{mL}^{[66]}$. 角 环素类天然产物是由 II 型聚酮合酶产生的芳香聚酮化 合物, 其结构中不同位置的糖基取代扩展了它们的结构 多样性. 角环素糖苷 72 73 对 A549、HepG2、MCF-7 和人鼻咽癌细胞 $\mathrm{CNE} 2$ 有很强的细胞毒性, 其中 73 比顺 铂的细胞毒活性高 20 倍, 其 $\mathrm{IC}_{50}$ 约为 $0.24 \sim 0.56$ $\mu \mathrm{mol} / \mathrm{L}^{[67]}$. 化合物 73 对正常肝细胞 HL7702 的 $\mathrm{IC}_{50}$ 值为 $3.67 \mu \mathrm{mol} / \mathrm{L}, 73$ 中的酮糖结构被认为可能是具有显著细 胞毒性的原因 ${ }^{[67]}$.马明等报道了从一株分离自印度洋 $3202 \mathrm{~m}$ 深的海底沉积物的链霉菌 PKU01297 中, 发现两 个新的角环素类化合物 74 75, 结构中的 1- $O-\beta-D$-吡喃 葡萄糖基团为首次在角环素类化合物中发现 ${ }^{[68]}$. 核苷 类化合物 77 对苏云金芽孢杆菌 BT01、W102 表现出较 强抗菌活性, MIC 分别为 $2.0 、 0.5 \mu \mathrm{g} / \mathrm{mL}$; 对白色念珠菌 ATCC 96901 和 CMCC (F) 98001 有一定的抗真菌活性, MIC 分别为 32 和 $8.0 \mu \mathrm{g} / \mathrm{mL}^{[69]}$. 马俊英等对链霉菌 SCSIO ZH16 进行基因组挖掘, 成功激活一个环肽基因 簇, 发现了 Atratumycin (78). 该化合物对结核分枝杆菌 $\mathrm{H} 37 \mathrm{Ra}$ 和 $\mathrm{H} 37 \mathrm{Rv}$ 的 $\mathrm{MIC}$ 分别为 3.8 和 $14.6 \mu \mathrm{mol} / \mathrm{L}^{[61]}$.

Butenolides 1 2 (82 83$)$ 、Ahpatin Ac (84)、Ahpatin $\operatorname{Pr}(\mathbf{8 5}) 、$ Fradiamines A $\sim \mathrm{B}(\mathbf{8 6} \sim \mathbf{8 7})$ 分别产自深海链霉菌 TP-A0873、ACT 232、MM456M-mF7, 这些链霉菌均分
离自日本海沉积物. 线性五肽 84 和 85 能有效抑制胃蛋 白酶和组织蛋白酶 B，而组织蛋白酶 B 是一个很有前途 的抗癌药物靶点 ${ }^{[64,70]}$. 铁载体 86 和 87 对艰难梭菌 BAA-1382 具有一定的抗菌活性，其 $\mathrm{IC}_{50}$ 分别为 32 和 8 $\mu \mathrm{g} / \mathrm{mL}^{[71]}$. Reyes 等从来自坎塔布里亚海的链霉菌 M-157 和 M-169 中, 分离出了 3-羟基喹啉羧酸 88 90、Anthracimycin B (91) 和 Anthracimycin. 化合物 90 对 HepG2 有轻微的细胞毒活性, 其 $\mathrm{IC}_{50}$ 为 $51.5 \mu \mathrm{mol} / \mathrm{L}^{[72]}$. 大环内 酯 91 抗菌活性优异，对四种革兰氏阳性菌 MSSA、 MRSA、VANS 和万古霉素敏感粪肠球菌的 MIC 分别为 $0.33 \sim 0.65 、 10.5 \sim 20.9 、 0.33 \sim 0.65 、 0.65 \sim 1.26 \mu \mathrm{mol} / \mathrm{L}$; 化合物 91 对革兰氏阴性菌大肠杆菌和肺炎克雷伯菌的 $\mathrm{MIC}$ 均大于 $41.8 \mu \mathrm{mol} / \mathrm{L}$. 根据此前的报道, 类似物 Anthracimycin 对所测的革兰氏阳性菌 MIC 均低于 0.03 $\mu \mathrm{g} / \mathrm{mL}^{[73]}$. 化合物 91 和 Anthracimycin 差异在于其 C-2 缺少甲基, 因此推测 Anthracimycin 中 C-2 甲基对其抗菌 活性至关重要用 ${ }^{[74]}$. 林双君等报道了从智利太平洋海 岸来源的链需菌 B9173 中分离出的含蒜醌的化合物 Flaviogeranins $B \sim D(92 \sim 94)$. 化合物 94 对金黄色葡萄 球菌和耻垢分枝杆菌的 MIC 为 $5.0 \sim 9.4 \mu \mathrm{g} / \mathrm{mL}$, 但对 A549 和 Hela 肿瘤细胞具有很强的细胞毒性, 其 $\mathrm{IC}_{50}$ 为 $0.2 \sim 0.8 \mu \mathrm{mol} / \mathrm{L}^{[75]}$.

\section{2 沙漠}

\section{1 沙漠栖息地}

地球陆地的五分之一被沙漠覆盖, 而缺乏植被和极 低的降雨量是沙漠最大的特征 ${ }^{[79]}$. 沙漠作为极端生态 环境，一般表现为高温干旱、紫外线辐射强和寡营养等 特征 ${ }^{[80]}$. Barberán 等 ${ }^{[81}$ 对来自美国亚利桑那州索诺兰沙

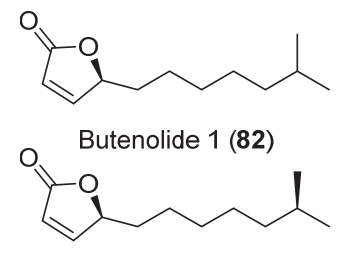

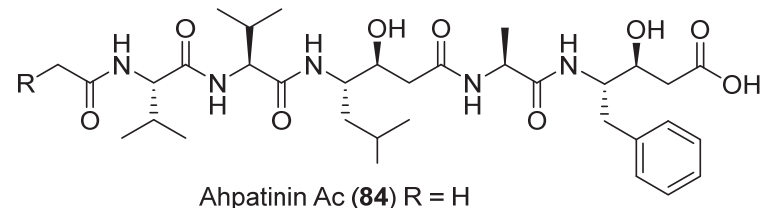
Ahpatinin $\operatorname{Pr}(85) \mathrm{R}=\mathrm{Me}$

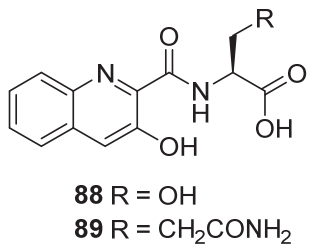<smiles>CC(=O)N(O)CCCCNC(=O)CC(CC(=O)O)(CC(=O)NCCCCNC(=O)CC(CC(=O)O)(CC(=O)NCCCCN(O)C(C)=O)C(=O)NCCCCN(O)C(C)=O)C(=O)O</smiles><smiles>CC1=CC[C@H]2C(C(=O)/C=C(\O)CC(=O)O[C@@H](C)/C=C\C=C/C(C)C)C=C[C@H]2C1</smiles>

Anthracimycin B (91)<smiles>CCOC1=C(C)C(=O)c2c(N)c(OC)cc(O)c2C1=O</smiles>

Flaviogeranin B1 (92)<smiles>COc1cc(O)c2c(c1N)C(=O)C(OC/C=C(\C)CCC=C(C)C)=C(C)C2=O</smiles>

Flaviogeranin B (93)<smiles>O=C(NC(CS)C(=O)O)c1coc(-c2nc3ccccc3cc2O)n1</smiles>

90<smiles>CC(C)=CCC/C(C)=C/CC1(O)C(=O)c2cc(O)c(C)c(O)c2C(=O)C1C(=O)O</smiles> 
漠西部的微生物进行了宏基因组分析后发现, 富营养和 寡营养地区来源的微生物在编码代谢途径、环境感知和 营养利用机制等多种功能基因上存在差异; 另外, 寡营 养地区来源微生物未注释基因占微生物群落总基因库 的 $80 \%$ 左右 ${ }^{[82-83]}$. Souza 等 ${ }^{[84]}$ 发现随着环境压力(营养匮 乏程度)的增加, 微生物之间的相互作用从竞争转变为 合作关系, 且随着环境压力的变化, 其次级代谢产物也 随之发生了变化. 从极度干旱的阿塔卡马沙漠土壤分离 的链霉菌 Leeuwenhoekii $\mathrm{C} 34^{\mathrm{T}}$ 包括 31 个生物合成基因

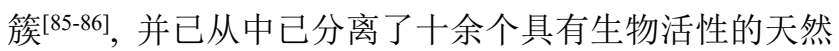
产物 ${ }^{[1,79,87-91]}$. 研究人员已从沙漠中发现丰富的放线菌 资源, 其中嗜热和嗜高温放线菌在沙漠土壤中分布最为 广泛, 超过其它放线菌 ${ }^{[48]}$. 这些放线菌的种类繁多, 所 产生的次级代谢产物较多 ${ }^{[92-93]}$. 目前研究较多的沙漠放 线菌主要来自于莫哈韦沙漠、蒙古沙漠 ${ }^{[93]}$ 、撒哈拉沙

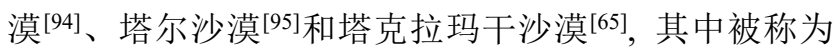
“死亡禁区” 的阿塔卡马沙漠较受关注 ${ }^{[96]}$. Alan 等 [47,97-98]对阿塔卡马沙漠进行微生物多样性研究时发现, 阿塔卡马沙漠富含放线菌资源, 大多数分离出的菌株属 于新的链霉菌菌种或稀有链雼菌属. Okoro 等 ${ }^{[99]}$ 报道从 阿塔卡马沙漠采集的土壤样品中分到的链霉菌菌株, 可 培养率高达 91\%。

\section{2 沙漠链霉菌中分离的新化合物}

Jaspars 等利用 OSMAC 方法, 发现从阿塔卡马沙漠 采集的链霉菌 C34 可以产生较罕见的 22 元大环内酯 Chaxalactins $\mathrm{A} \sim \mathrm{C}(\mathbf{9 5} \sim 97)^{[79]}$ 和安沙霉素型聚酮 Chaxa-
Mycins $A \sim D(98 \sim 101)^{[90]}$. 化合物 95 97 对革兰氏阳 性菌具有较强的抗菌活性，它们对金黄色葡萄球菌 MIC 低于 $1 \mu \mathrm{g} / \mathrm{mL}$ (表 2), 对李斯特菌和枯草芽孢杆菌 MIC 为 $3 \sim 6 \mu \mathrm{g} / \mathrm{mL}$. 化合物 101 对金黄色葡萄球菌 ATCC 25923 的 MIC 为 $0.05 \mu \mathrm{g} / \mathrm{mL}$ ，对耐药金黄色葡萄球菌的 MIC 低于 $1 \mu \mathrm{g} / \mathrm{mL}$. Imhoff 等对同样来自于阿塔卡马沙 漠的链霉菌 C38 和 DB634 进行发酵, 获得 22 元大环内 酯 Atacamycins $\mathrm{A} \sim \mathrm{C}(\mathbf{1 0 2} \sim \mathbf{1 0 4})^{[91]}$ 和氨基醌 Abenquines $\mathrm{A} \sim \mathrm{D}(\mathbf{1 0 5} \sim \mathbf{1 0 9})^{[89]}$. 化合物 $102 \sim 104$ 和 105 、 109 对磷酸二酯酶表现出一定的抑制作用, $\mathrm{EC}_{50}$ 分别为 $1.30 \sim 4.07$ 和 $3.9 \sim 4.8 \mu \mathrm{mol} / \mathrm{L}$. 化合物 $102 \sim 103$ 对 42 种不同的人肿瘤细胞的细胞毒性均有一定的抑制作用, $\mathrm{IC}_{50}$ 的平均值为 13.4 和 $20.4 \mu \mathrm{mol} / \mathrm{L}$; 化合物 102 对结肠 癌、乳腺癌和子宫癌细胞的 $\mathrm{IC}_{50}$ 在 $2.66 \sim 5.93 \mu \mathrm{mol} / \mathrm{L}$, 104 对结肠癌细胞的 $\mathrm{IC}_{50}$ 为 $8.51 \mu \mathrm{mol} / \mathrm{L}$.

Rateb 等[87]通过对链霉菌 C34 和真菌 MR2012 共培 养, 产生了 Luteoride D (110)和 Pseurotin G (111), 这两 个化合物均无明显的抑菌活性或细胞毒性. 对阿塔卡马 沙漠来源的链霉菌 C58 和 KNN42.F 进行发酵, 得到了 Asenjonamides $\mathrm{A} \sim \mathrm{C}(\mathbf{1 1 2} \sim \mathbf{1 1 4})$ 和套索肽 Chaxapeptin (115). 化合物 112 114 对金黄色葡萄球菌、枯草芽孢杆 菌、大肠杆菌、粪肠球菌和耻垢分枝杆菌具有较显著的 抗菌活性, 其 $\mathrm{MIC}$ 分别为 $1.8 \sim 3.6 、 1.7 \sim 3.9 、 5.4 \sim 17.3$ 、 $3.9 \sim 13.7$ 和 $10.3 \sim 19.1 \mu \mathrm{g} / \mathrm{mL}^{[96]}$.

新型套索肽 115 在人肺癌细胞株 A549 的细胞侵袭 实验中表现出明显的抑制活性; 对金黄色葡萄球菌和枯

表 2 沙漠链霉菌的新天然产物

Table 2 New compounds from desert-derived Streptomyces

\begin{tabular}{|c|c|c|c|c|}
\hline Compd. & Source & Activity (MIC or $\mathrm{IC}_{50}$ ) & Region & Time \\
\hline Chaxalactins $\mathrm{A} \sim \mathrm{C}(\mathbf{9 5} \sim 97)$ & $\mathrm{C} 34$ & $\begin{array}{l}\text { S. aureus: }<1 \mu \mathrm{g} / \mathrm{mL} \text { L. monocytogenes/ } \\
\text { B. subtilis: } 3 \sim 6 \mu \mathrm{g} / \mathrm{mL}\end{array}$ & Atacama & $2011^{[79]}$ \\
\hline Chaxamycins $\mathrm{A} \sim \mathrm{D}(\mathbf{9 8} \sim 101)$ & $\mathrm{C} 34$ & $\begin{array}{l}\text { S. aureus ATCC 25923: } 0.05 \mu \mathrm{g} / \mathrm{mL} \text { (101) } \\
\text { MRSA: }<1 \mu \mathrm{g} / \mathrm{mL} \text { (101) }\end{array}$ & Atacama & $2011^{[90]}$ \\
\hline Atacamycins $\mathrm{A} \sim \mathrm{C}(\mathbf{1 0 2} \sim \mathbf{1 0 4})$ & $\mathrm{C} 38$ & $\begin{array}{l}\text { R.solanacearum } / \mathrm{PDE}-4 \mathrm{~B} 2 \text { Enzyme inhibitor: } \\
1.30 \sim 4.07 \mu \mathrm{mol} \cdot \mathrm{L}^{-1} / \text { antiproliferative }\end{array}$ & Atacama & $2011^{[91]}$ \\
\hline Abenquines $\mathrm{A} \sim \mathrm{D}(\mathbf{1 0 5} \sim \mathbf{1 0 9})$ & DB634 & Enzyme inhibitor for phosphodiesterase type $4 b$ & Atacama & $2011^{[89]}$ \\
\hline Luteoride D (110)/Pseurotin G (111) & $\mathrm{C} 34+\mathrm{MR} 2012$ & - & Atacama & $2017^{[115]}$ \\
\hline Asenjonamides $\mathrm{A} \sim \mathrm{C}(\mathbf{1 1 2} \sim \mathbf{1 1 4})$ & KNN 42.f & $\begin{array}{l}\text { S. aureus/B. subtilis/E. coli/E. faecalis/M. } \\
\text { smegmatis: } 1.8 \sim 17.3 \mu \mathrm{g} / \mathrm{mL}\end{array}$ & Atacama & $2018^{[96]}$ \\
\hline Chaxapeptin (115) & $\mathrm{C} 58$ & Inhibitory activity against A549 & Atacama & $2015^{[100]}$ \\
\hline Leepeptin (116) & $\mathrm{C} 34^{\mathrm{T}}$ & - & Atacama & $2019^{[101]}$ \\
\hline Huascopeptin (117) & $\mathrm{HST} 28^{\mathrm{T}}$ & - & Atacama & $2020^{[102]}$ \\
\hline 118 & WAB9 & Pseudomonas aeruginosa IPA1: $10 \mu \mathrm{g} / \mathrm{mL}$ & Saharan & $2015^{[103]}$ \\
\hline Pyridine-2,5-diacetamide (119) & DA3-7 & $\begin{array}{l}\text { E. coli ATCC 10536/C.neoformans ATCC } \\
\text { 90113: } 31.25 \mu \mathrm{g} / \mathrm{mL}\end{array}$ & Saudi Arabian & $2018^{[104]}$ \\
\hline Grincamycins $L \sim N(120 \sim 122)$ & XZHG99 T & $\begin{array}{l}\text { A549/H157/MCF-7/MDA-MB-231/ } \\
\text { HepG2: } 1.92 \sim 9.12 \mu \mathrm{mol} \cdot \mathrm{L}^{-1}\end{array}$ & Color desert & $2018^{[69]}$ \\
\hline
\end{tabular}


<smiles>[R2]OC(/C=C/C=C/CC(/C=C/CC)OC(=O)/C=C/C(C)=C/C=C/C(C)C/C=C\C(C)C(O)C([R7])[R2])C(C)O</smiles>

Chaxalactin A (95) $\mathrm{R}^{1}=\mathrm{H}, \mathrm{R}^{2}=\mathrm{H}$ Chaxalactin $B(96) R^{1}=O H, R^{2}=H$ Chaxalactin $\mathrm{C}(97) \mathrm{R}^{1}=\mathrm{OH}, \mathrm{R}^{2}=\mathrm{Me}$

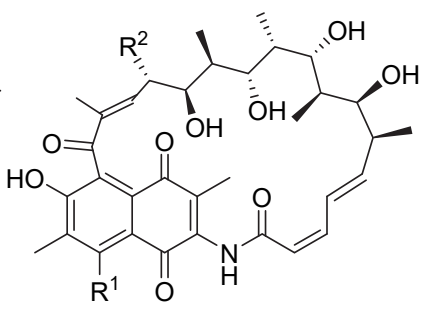

Chaxamycin $\mathrm{A}(98) \mathrm{R}^{1}=\mathrm{OH}, \mathrm{R}^{2}=\mathrm{Me}$

Chaxamycin $B(99) R^{1}=H, R^{2}=M e$

Chaxamycin $\mathrm{C}(\mathbf{1 0 0}) \mathrm{R}^{1}=\mathrm{OH}, \mathrm{R}^{2}=\mathrm{CH}_{2} \mathrm{OH}$

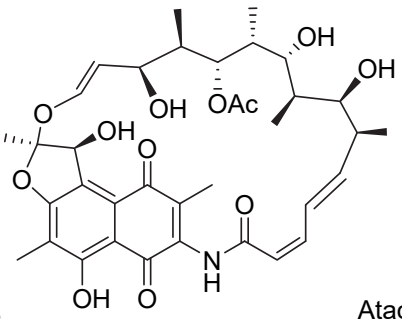<smiles>[R]C(C)C([R])/C=C/C(C)C(O)C([R])C(/C=C\C=C/C(C)=C\C=C/C(=O)OC(/C=C/C)(/C=C/C)CC)OC</smiles><smiles>C=C(C)NC1CC(=O)C(NC(Cc2ccccc2)C(=O)O)=CC1=O</smiles>

Abenquine A (105)<smiles>CC(=O)NC1=CC(=O)C(N[C@@H](CC(C)C)C(=O)O)=CC1=O</smiles>

Abenquine B1 (106)<smiles>CCC(C)C(NC1=CC(=O)C(NC(C)=O)=CC1=O)C(=O)O</smiles>

Abenquine B2 (107)<smiles>CC(=O)NC1=CC(=O)C(NC(C(=O)O)C(C)C)=CC1=O</smiles>

Abenquine $C$ (108)
Atacamycin $A\left(\right.$ 102) $\mathrm{R}^{1}=\mathrm{OMe}, \mathrm{R}^{2}=\mathrm{OH}$ Atacamycin $B\left(\right.$ 103) $R^{1}=H, R^{2}=\mathrm{OH}$ Atacamycin $\mathrm{C}(\mathbf{1 0 4}) \mathrm{R}^{1}=\mathrm{H}, \mathrm{R}^{3}=\mathrm{H}$<smiles>C=CCOCCOCCOCCOC</smiles><smiles>CCCCC=CC(C)C(=O)C(C)C(=O)NCCO</smiles>

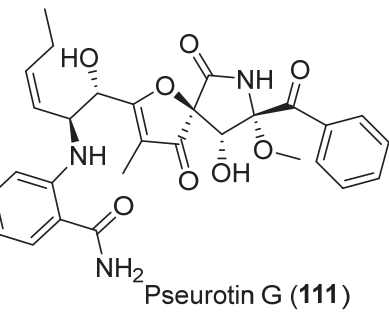<smiles>C=CC=CCCCC(NC(=O)C(C)CO)C(=O)N1CCCC1C=O</smiles><smiles>CNC1=CC(=O)C(N[C@@H](Cc2c[nH]c3ccccc23)C(=O)O)=CC1=O</smiles>

$$
\text { Abenquine D (109) }
$$<smiles>CCCC/C=C(\C)C(=O)C(C)C(N)=O</smiles>

Asenjonamide B (113)

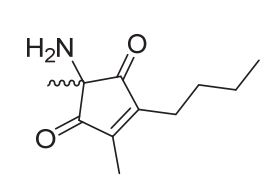

Asenjonamide C (114)<smiles>CCNC(=O)CN</smiles>

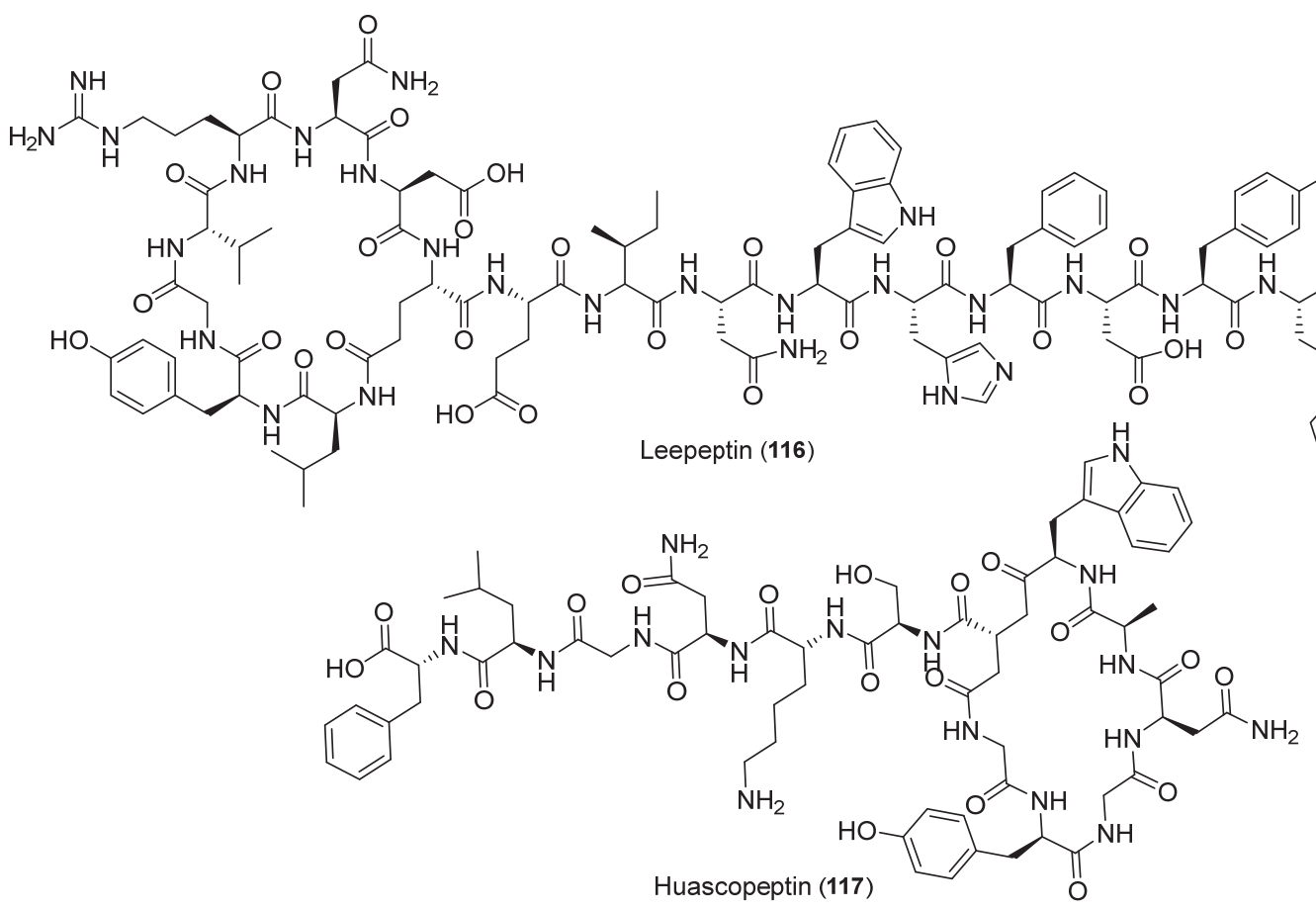<smiles>CC(C)CC(NC(=O)C(CC(N)=O)NC(=O)C(Cc1ccccc1)NC(=O)C(Cc1ccccc1)NC(=O)C(Cc1ccccc1)NC(=O)O)C(=O)O</smiles>

Chaxapeptin (115)

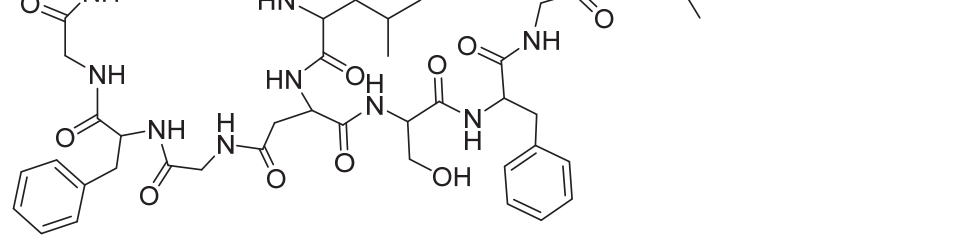

Huascopeptin (117) 
草芽孢杆菌的抗菌活性较弱, 其 MIC 为 $30 \sim 35$ $\mu \mathrm{g} / \mathrm{mL}^{[100]}$. 对链需菌属 leeuwenhoekii $\mathrm{C} 34 \mathrm{~T}$ 的基因组序 列进行生物合成基因簇分析, 发现该菌株中含有套索肽 基因簇, 但是尚未合成套索肽. 该套索肽的生物合成基 因簇在天蓝色链霉菌中进行异源表达后, 得到了一个新 的套索肽 Leepeptin (116) ${ }^{[101]}$. Asenjo 等 ${ }^{[102]}$ 从阿塔卡马沙 漠的盐沼分离的链霉菌菌株 HST28T 中, 发现了含有 13 个氨基酸残基的 II 型套索肽 Huascopeptin (117). 该套索 肽含有一个新型的七元大环内酰胺, 是迄今为止分离到 的最小的套索肽, 而其套索结构由基于 NOE 约束的分 子动力学实验证实.

Sabaou 等 ${ }^{[103]}$ 从来自阿尔及利亚撒哈拉沙漠土壤中 的链霉菌 WAB9 中, 分得一种新型的含有羟肜酸官能团 的化合物 118. 该化合物对革兰氏阳性菌和阴性菌、丝 状真菌和酵母均表现出一定的抗菌活性. Dhanasekaran 等 ${ }^{[104]}$ 从沙特阿拉伯沙漠中分离出来的链霉菌 DA3-7 中, 发现了一种新的吡啶生物碱 119. 该化合物对大肠杆菌 和新生隐球菌具有微弱的抗菌活性, 其 MIC 为 31.25 $\mu \mathrm{g} / \mathrm{mL}$. 张华等 ${ }^{[69]}$ 从西藏登巴彩色沙漠采集的土壤样本 中发现链霉菌 XZHG99T, 从中分离出新的角环素糖苷 Grincamycins $\mathrm{L} \sim \mathrm{N}(\mathbf{1 2 0} \sim 122)$. 化合物 119 121 对人 癌细胞 A549、H157、MCF-7、MDA-MB-231 和 HepG2 有一定的细胞毒性, $\mathrm{IC}_{50}$ 为 $1.92 \sim 9.12 \mu \mathrm{mol} / \mathrm{L}$.<smiles>NC(Cc1ccccc1)C(=O)N(O)C(=O)C(N)Cc1ccccc1</smiles><smiles>CC(=O)Nc1ccccc1</smiles>

118

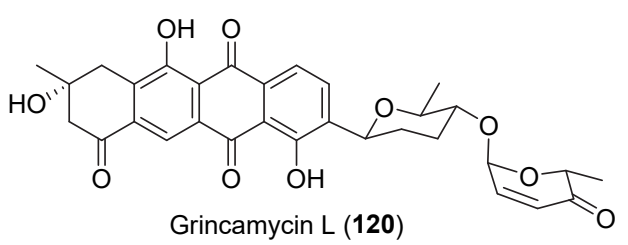<smiles>[R]c1ccc2c(c1O)C(=O)c1cc(OC)c3c(c1C2=O)C(=O)CC(C)(O)C3</smiles><smiles>[R]c1ccc2c(c1O)C(=O)c1cc3c(O)cc(C)cc3c(O)c1C2=O</smiles>
Grincamycin N (122)

Grincamycin M (121)

\section{3 火山}

\section{1 火山沉积物}

火山沉积物在空气中风化后, 会形成水铝英石、水 铁矿等矿物质. 这些特殊的矿物质及其形成的腐殖质使 火山沉积物区别于其它生态环境中的土壤, 因此火山沉

积物也属于特殊的生态环境 ${ }^{[105]}$. 最近，人们发现火山 沉积物中的微生物与其它环境中的微生物具有独特的 遗传特征, 并能够产生新颖的天然产物 ${ }^{[106-108]}$. Kelly 等 ${ }^{[109]}$ 从冰岛埃亚菲亚德拉冰盖火山的玄武火山岩中分 离得到了一系列微生物, 并通过 $16 \mathrm{~S}$ rRNA 基因序列测 序后将其归属为放线菌及变形杆菌. Goodfellow 等 ${ }^{[110]}$ 从 埃特纳火山沉积物中分离得到了 140 个微生物菌株, 发 现有 26 个属于放线菌门. Kay 等 ${ }^{[111]}$ 在加拿大赫尔克肯 瀑布火山洞穴中分离得到了 119 株放线菌. 对这些放线 菌的发酵液进行抗幼虫芽孢杆菌生物活性测试, 得到一 株对幼虫芽狍杆菌具有显著抗菌活性的放线菌 $\mathrm{E} 9$, 进 一步通过 $16 \mathrm{~S}$ rRNA 将其归属为链霉菌.

\section{2 火山沉积物链霉菌中分离的新化合物}

济州岛是韩国的一个亚热带火山岛, 其火山沉积物 中富含丰富的玄武岩、粗面岩、长石和镁铁矿物, 明显 有别于其它士壤样品. Oh 等[112]从韩国济州岛海岸分离 得到一株链霉菌 SNJ042, 发现该菌株产生 Ohmyungsamycins $A \sim B(123 \sim 124)$ 两种新型环肽. 它们由 10 个 氨基酸组成, 其中有 2 个非天然氨基酸, 4 个氮-甲基化 氨基酸以及一个双肽侧链.

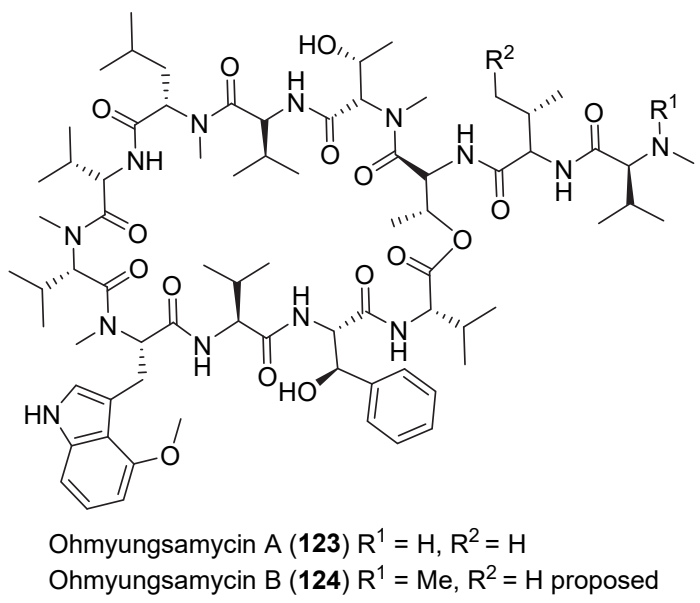

Suh 等[113]通过全合成制备了 $123 \sim 124$ 及其衍生物，并 将 124 的结构进行了修订. 在此基础上, 发现其环肽结 构是 Ohmyungsamycins 具有优异抗结核分枝杆菌活性 的原因, 其对结核分枝杆菌 H37Rv 的 MIC 分别为 33.3 和 $108.3 \mathrm{nmol} / \mathrm{L}$ (表 3 ). 化合物 123 具有广谱的抗菌活性. Ohmyungsamycins 通过腺苷酸活化蛋白激酶依赖信号, 激活抗菌自噬并抑制结核分枝杆菌感染期间的过度炎 症反应，是一种很有前景的新型抗结核分枝杆菌治疗药 物 ${ }^{[114]}$. 化合物 123 对 HCT-116、A549、SNM-638、 MDA-MB-231 和 SKHEP-1 等癌细胞株具有优异的细胞 毒性, $\mathrm{IC}_{50}$ 为 $359 \sim 816 \mathrm{nmol} / \mathrm{L}$. 化合物 124 的细胞毒性 较弱, 其 $\mathrm{IC}_{50}$ 为 $12.4 \sim 16.8 \mu \mathrm{mol} / \mathrm{L}$, 其原因可能在于其 结构中含有 $N, N$-二甲基顺氨酸. 
表 3 来自火山链霉菌的新化合物

Table 3 Volcanic Streptomyces-derived new compounds

\begin{tabular}{|c|c|c|c|c|}
\hline Compd. & Streptomyces $s p$. & Activity (MIC or $\mathrm{IC}_{50}$ ) & Region & Time \\
\hline Ohmyungsamycins A/B (123/124) & SNJ042 & $\begin{array}{l}\text { M. tuberculosis } \mathrm{H} 37 \mathrm{Rv}: 33.3 / 108.3 \\
\mathrm{nmol} \cdot \mathrm{L}^{-1}, \mathrm{HCT}-116 / \mathrm{A} 549 / \mathrm{SNM}-638 / \\
\text { MDA-MB-231: } 359 \sim 816 \mathrm{nmol} \cdot \mathrm{L}^{-1}(\mathbf{1 2 3})\end{array}$ & Korean volcanic & $2013^{[112]}$ \\
\hline Pontemazines A/B (125/126) & MT1123 & $\begin{array}{l}\text { Protective effect on HT- } 22 \text { mouse } \\
\text { hippocampal neuronal cells }\end{array}$ & Korean volcanic & $2015^{[111]}$ \\
\hline Ulleungdin (127) & KCB13F003 & Inhibitory activity against A549 & Korean volcanic & $2018^{[96]}$ \\
\hline Donghaesulfins A/B (128/129) & SMD119 & $\begin{array}{l}\text { Induced quinone reductase activity/ } \\
\text { antiangiogenesis }\end{array}$ & Korean volcanic & $2019^{[116]}$ \\
\hline Donghaecyclinones $\mathrm{A} \sim \mathrm{C}(\mathbf{1 3 0} \sim \mathbf{1 3 2})$ & SUD119 & $\begin{array}{l}\text { HCT116/MDA-MB231/SNU638/A549/ } \\
\text { SK-HEP1: } 6.0 \sim 28.9 \mu \mathrm{nmol} \cdot \mathrm{L}^{-1}\end{array}$ & Korean volcanic & $2020^{[117]}$ \\
\hline
\end{tabular}

苯甲酰胺吩嗪衍生物 Pontemazines $\mathrm{A} \sim \mathrm{B}(\mathbf{1 2 5} \sim$ 126)从链霉菌 MT1123 分离获得, 在对谷氨酸诱导的小 鼠海马 HT-22 细胞损伤中, 对神经元细胞具有保护作 用 ${ }^{[80]}$. Ahn 等 ${ }^{[118]}$ 从韩国 Mlleung 火山岛的链霉菌 $\mathrm{KCB} 13 \mathrm{~F} 003$ 分离出一种含 15 个氨基酸的 II 类套索肽 Ulleungdin (127). 套索肽 127 对 A549 细胞的侵袭和迁 移具有明显的抑制作用, 而肽链长度或大内酰胺环的大 小会影响其活性. Bae 等从源于韩国 Mlleung 火山岛的 链霉菌 SMD119 和 SUD119 中, 分离到由硫醚键连接的 角环素二聚体 Donghaesulfins $A \sim B(128 \sim 130)^{[116]}$ 和 Donghaecyclinones $\mathrm{A} \sim \mathrm{C}(\mathbf{1 3 0} \sim \mathbf{1 3 2})$. 化合物 128 可以 诱导产生醌还原酶, 而 130 表现出抗血管生成活性. 化 合物 130 132 对多种人癌细胞 HCT116、MDA-MB231、 SNU638、A549、SK-HEP1 具有一定的细胞毒性; 其中
132 的 $\mathrm{IC}_{50}$ 为 $6.7 \sim 9.6 \mu \mathrm{mol} \cdot \mathrm{L}^{-1}$. 角环素二聚体 $128 \sim$ 129 并无显著的细胞毒性, 这说明 C-3'的立体化学对其 细胞毒性很重要 ${ }^{[117]}$.

\section{4 极地}

\section{1 极地生态环境}

南极和北极以寒冷、干燥及强紫外辐射而闻名, 科 研人员从冰芯、雪样、土壤沉积物、岩石和海洋生物等 各类样品中发现了大量微生物, 其中以嗜冷、耐冷微生 物为优势微生物. 高盐(海冰、盐湖)、高温(火山口)、酸 性(湖泊)及高压(深海、深冰芯)等不同生境的存在，极大 地丰富了极地的微生物资源. 南极和北极因其独特的地 理位置及严酷的自然环境条件, 还未受到污染并保留了 其原始状态 ${ }^{[119]}$.<smiles>[R1]C(=O)c1ccccc1NCc1ccc(OC)c2nc3c(C([R1])=O)cccc3nc12</smiles>

Pontemazine A (125) $\mathrm{R}^{1}=\mathrm{R}^{2}=\mathrm{NH}_{2}$ Pontemazine $\mathrm{B}\left(\right.$ 126) $\mathrm{R}^{1}=\mathrm{OH}, \mathrm{R}^{2}=\mathrm{NH}_{2}$

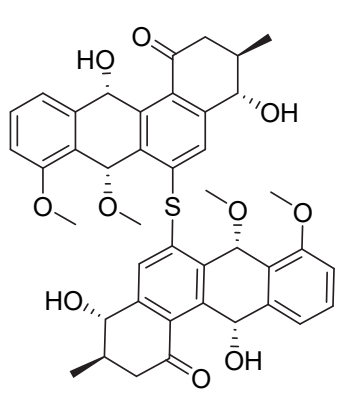

Donghaesulfin A (128)

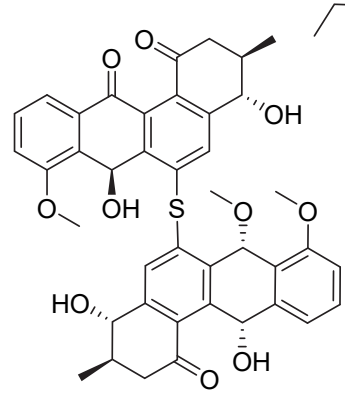

Donghaesulfin B (129)<smiles>CCNC(=O)CC(NC(=O)C(CCCCN)NC(=O)CNC(=O)C(Cc1c[nH]c2ccccc12)NC(=O)CC)C(=O)NC(C(=O)NC(Cc1ccccc1)C(=O)NCC(=O)NC(Cc1cnc[nH]1)C(=O)NC(Cc1ccc(O)cc1)C(=O)NCC(=O)NCC(=O)O)C(C)CC</smiles><smiles>CCC(C)C(NC(=O)C(Cc1ccccc1)NC(=O)CN)C(=O)NCC(C)=O</smiles><smiles>COc1cccc2c1C1OCC2c2c(ccc3c2C(=O)CC(C)[C@H]3O)O1</smiles>

Donghaecyclinone A (130)<smiles>COc1cccc2c1C(=O)O[C@H]2c1c(O)ccc2c1C(=O)C[C@@H](C)[C@H]2O</smiles>

Donghaecyclinone B (131)<smiles>COc1cccc2c1C(=O)O[C@H]2c1c(O)ccc2c1C(=O)CC(C)[C@H]2O</smiles>

Donghaecyclinone C (132) 
极地特殊生境导致了大量极地微生物在其基因组 成、酶学特性以及代谢调控等方面的独特性. 近十多年 来的研究显示, 极地微生物在特殊环境(尤其是低温)适 应机制、新药开发、新型酶制剂研制、新型保健食品开 发等方面具有广阔的前景. Pasupuleti 等[120]发现从极地 生物(包括原核生物和真核生物)中获得的 $38 \%$ 的天然产 物有一定的药用价值. 田元等对 2001 2017 年从北极 和南极微生物、地衣、苔藓和海洋动物中发现的 219 种 新型天然产物及其合成的衍生物进行了总结 ${ }^{[121]}$. Melo

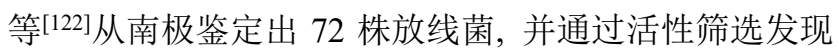
了 17 株有潜力的菌株, 而其中链霉菌 CMAA 1527 和 CMAA 1653 的粗提物具有显著的抗增殖活性.

\section{2 从极地链霉菌分离出的新天然产物}

林文翰等对来自北极的链霉菌 Nitrosporeus CQT14-24 和 YBH10-5 进行发酵分离, 分别得到 2 个新 的生物碱 Nitrosporeusines $A \sim B(133 \sim 134)^{[123]}$ 和 7 个新 化合物 Nitrosporeunols $A \sim G(137 \sim 143)^{[124]}$. 在浓度为 $50 \mu \mathrm{mol} / \mathrm{L}$ 时, 133 134 对 MDCK 细胞中流行性感冒 WSN 病毒(H1N1)的抑制活性分别达到 18.6\%和 30.9\% (表 4). 在体外血小板减少实验中, 随着 134 的剂量增加 A/WSN/33 流感病毒感染的 MDCK 细胞产生的子代病 毒减少; 在抑制病毒斑块形成的实验中, 134 的抗病毒 活性与磷酸奥司他韦相当. Reddy 等 ${ }^{[125]}$ 以环戊烷并 $1,3-$ 二酮吡咯和对羟基苯甲酸为底物五步反应便能高效立 体选择性合成 133 和 134 以及其对映异构体. 裴月湖从
南极海洋来源的链霉菌 S. cavourensis YY01-17 分离到 两个新化合物 $135 \sim 136^{[124]}$.

$\mathrm{Oh}$ 等[126]从一株北极海洋链霉菌 ART5 中分离出具 有苯并噁嗪环结构的 Arcticoside (144)和 6-氯取代的芳 构化的烯二炔 C-1027 发色团 145, 以及已知 C-1027 发 色团和 Fijiolides A 和 B. 化合物 144 和 145 细胞毒性显 著, $\mathrm{IC}_{50}$ 分别为 0.9 和 $2.7 \mu \mathrm{mol} / \mathrm{L}$. 樊成奇等 ${ }^{[127]}$ 从极地海 洋链霉菌 $623 \mathrm{~F}$ 和 NJES13 中获得了四个新的芳香酮类 化合物 146 149, 化合物 149 属于角环素类化合物 ${ }^{[128]}$. $\mathrm{Nam}$ 等 ${ }^{[129]}$ 从南极链霉菌 SCO736 中分离到一种新的齐 扎烷型倍半萜 Antartin (150), 该化合物对 A549、H1299 和 U87 等癌细胞株有一定的细胞毒性, 可使细胞周期阻 滞在 G1 期. 朱伟明等 ${ }^{[130-131]}$ 从南极链霉菌 OUCMDZ4348 分离得到大环内酰胺类化合物 Cyclamenols $B \sim F$ $(151 \sim 155)$, 并提出了分子内 Diels-Alder 反应的生物合 成途径. 化合物 151 和 154 对胃癌 N87 细胞株具有选择 性的抑制作用, $\mathrm{IC}_{50}$ 分别为 10.8 和 $9.8 \mu \mathrm{mol} \cdot \mathrm{L}^{-1}$.

\section{5 结论与展望}

据不完全统计，研究人员在 $2009 \sim 2020$ 年 8 月，从 深海、沙漠、火山和极地链霉菌中共分离出 155 个新天 然产物. 对深海链霉菌中天然产物的发现正稳步发展, 而对沙漠、火山和极地来源链霉菌天然产物的发现才刚 起步(图 1). 产生新天然产物最多的链霉菌来源依次是 深海(94 个)、沙漠(28 个)、极地(23 个)、火山(10 个), 分

表 4 极地链霉菌的新天然产物

Table 4 New compounds from polar-derived Streptomyces

\begin{tabular}{|c|c|c|c|c|}
\hline Compd. & Source & Activity (MIC or IC50) & Region & Time \\
\hline Nitrosporeusines A/B (133/134) & Nitrosporeus CQT14-24 & Inhibit H1N1 virus & Arctic & $2013^{[123]}$ \\
\hline $135 / 136$ & Cavourensis YY01-17 & - & Antarctic & $2013^{[124]}$ \\
\hline Nitrosporeunols $A \sim G(\mathbf{1 3 7} \sim \mathbf{1 4 3})$ & nitrosporeus YBH10-5 & - & Arctic & $2014^{[132]}$ \\
\hline $\begin{array}{l}\text { Arcticoside }(\mathbf{1 4 4 )} \\
\text { C-1027 chromophore-V (145) }\end{array}$ & ART5 & $\begin{array}{l}\text { MDA-MB231/HCT-116: } \\
0.9 \sim 2.7 \mu \mathrm{mol} \cdot \mathrm{L}^{-1}(\mathbf{9 4})\end{array}$ & Arctic & $2014^{[126]}$ \\
\hline $146 \sim 148$ & $623 \mathrm{~F}$ & - & Arctic & $2015^{[127]}$ \\
\hline 149 & NJES13 & - & Antarctic & $2015^{[128]}$ \\
\hline Antartin (150) & $\mathrm{SCO} 736$ & A549/H1299/U87 & Antarctic & $2018^{[133]}$ \\
\hline Cyclamenols $B \sim D(151 \sim 153)$ & OUCMDZ-4348 & N87: $10.8 \mu \mathrm{mol} \cdot \mathrm{L}^{-1}(\mathbf{1 5 1})$ & Antarctic & $2019^{[130]}$ \\
\hline Cyclamenols $\mathrm{E} \sim \mathrm{F}(\mathbf{1 5 4} \sim \mathbf{1 5 5})$ & OUCMDZ-4348 & $\mathrm{N} 87: 9.8 \mu \mathrm{mol} \cdot \mathrm{L}^{-1}(\mathbf{1 5 4})$ & Antarctic & $2020^{[131]}$ \\
\hline
\end{tabular}<smiles>O=C(SC12CCC(O)C1C(=O)NC2=O)c1ccc(O)cc1</smiles>

Nitrosporeusines A (133)<smiles>O=C1NC(=O)C2(C(=O)Sc3ccc(O)cc3)CC[C@@H](O)[C@@H]12</smiles>

Nitrosporeusines B (134)<smiles>CC(O)C(=O)OC(C)C(C)O</smiles>

135<smiles>CC/C=C(\C)C(O)C(C)C(N)=O</smiles>
$\mathrm{HO}$<smiles>OCc1ccccc1P</smiles>

136

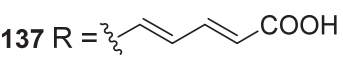

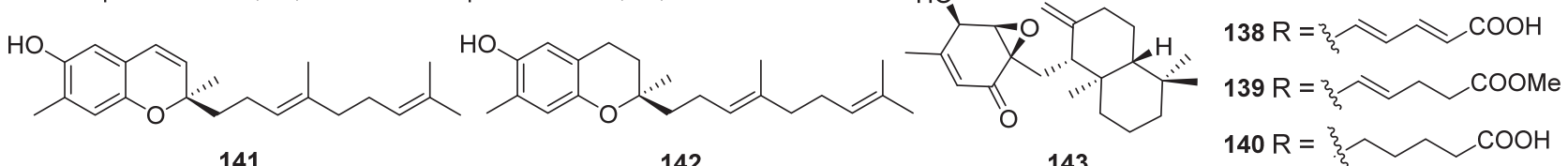

142
143 


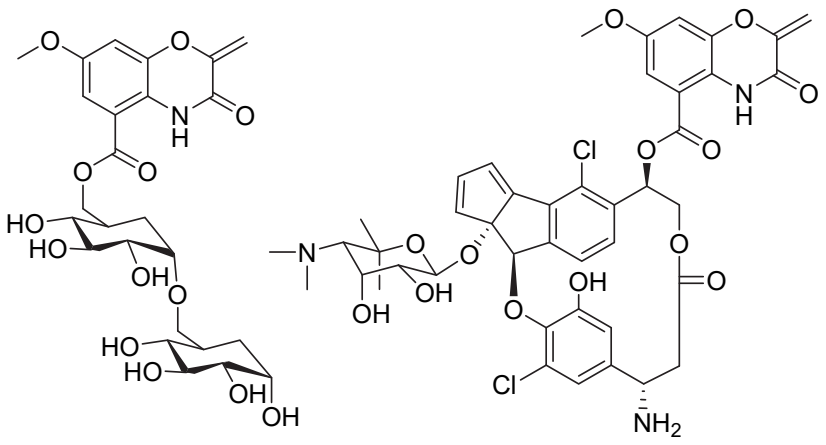

Arcticoside (144)

C-1027 chromophore-V (145)

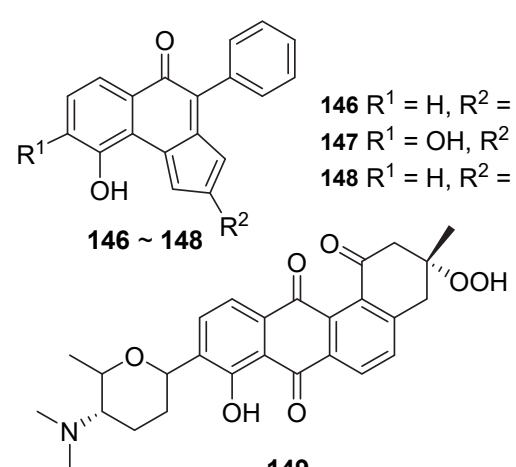

149<smiles>CCC(C)/C=C/C=C/[C@H]1C=C[C@H]2C(O)CC=C[C@H]2[C@H]1/C=C/C(=O)NC(=O)C(C)CC(C)C</smiles>

Cyclamenol D (153)<smiles>CC(C)/C=C/C=C/C(=O)NCC(C)/C=C\C=C\C=C\[C@H]1[C@H](O)[C@H](O)C[C@H]1O</smiles>

Cyclamenol E (154)<smiles>C/C=C\CC(C)/C=C\C=C/C=C/[C@H]1[C@@H](O)CC[C@H]1/C=C/C=C/C(=O)NCC(C)O</smiles>

$\overline{\mathrm{O}} \mathrm{H}$

Cyclamenol F (155)
Cyclamenol C (152)

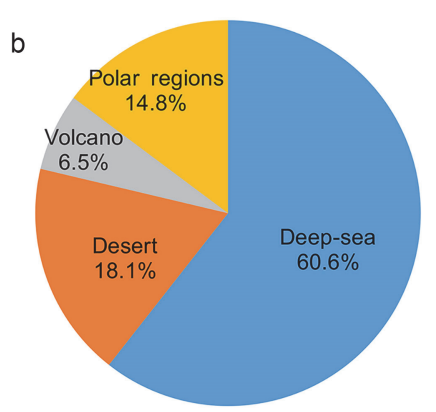

d No Bioactivity

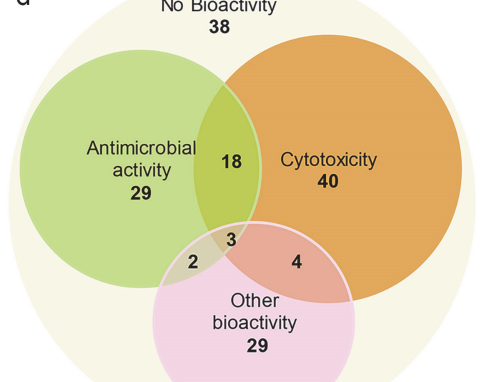

Polyketides alkaloids peptides others

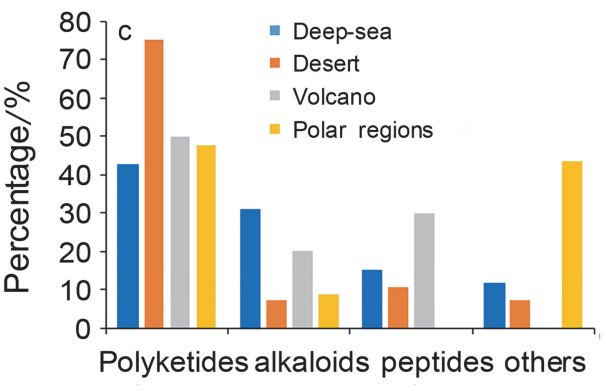

图 12009 年到 2020 年从深海、沙漠、火山和极地来源的链霉菌产生的新天然产物的分析

Figure 1 Analysis of new natural products produced by Streptomyces strains isolated from deep-sea, desert, volcano sediments, and polar regions

(a) The number of new natural products isolated each year; (b) the ratio of new natural products from deep-sea-, desert-, volcano- and polar region-derived Streptomyces; (c) the ratio of new natural products based on structures; (d) the ratio of bioactive natural products

别占 $60.6 \% 、 18.1 \% 、 14.8 \%$ 和 $6.5 \%$ (图 1B). 我国对深海 来源的链霉菌天然产物发现上占据了重要地位, 贡献了 74 个新天然产物, 占深海链需菌来源的新天然产物 $78.7 \%$. 在这 74 个化合物中, 有 49 个是从我国南海来源 链霉菌中分离得到, 标志我国海洋天然产物的研究具有 一定的国际影响力. 沙漠来源链霉菌中分离的新天然产
物主要集中在阿塔卡马沙漠. 在 2009 2020 年 8 月期间 分离的 28 个新天然产物中, 只有 2 个由我国科学家从西 藏彩色沙漠来源的链霉菌中获得. 从火山沉积物发现的 链霉菌中分离出新天然产物的研究较少, 过去分离出的 新天然产物全部来自于韩国的火山岛屿. 火山沉积物和 极地来源链霉菌中分离出的天然产物数量不多, 但它们 


\section{具有多种生物活性.}

对沙漠、火山和极地这三个极端生态环境来源的链 霉菌天然产物较少, 而深海链霉菌来源的天然产物结构 较为多样. 其结构类型包括聚酮、生物碱、类萜、肽、 卤代物、糖苷等, 其中聚酮(77 个)、生物碱(35 个)、肽 (20 个)分别占化合物总数的 $49.7 \% 、 22.6 \%$ 和 $12.9 \%$. 在 这 155 个天然产物中, 有 117 个(约 75.4\%)有一定的生物 活性，如细胞毒性、抗菌活性和自由基清除等(图 1D); 有 65 个化合物对肿瘤细胞有一定的细胞毒活性, 52 个 化合物有抑菌作用, 分别占活性化合物总数的 $55.5 \%$ 和 44.4\%; 其它 27 个化合物具有多种生物活性. 上述分析 说明来自于极端环境的链霉菌是发现新天然产物和先 导化合物的重要来源之一. 另外, 高通量 DNA 测序技 术极大地方便了链霉菌的全基因组测序, 加速了对其产 生的天然产物生物合成研究. 由于链霉菌的遗传转移系 统的建立相对成熟, 结合在模式链霉菌中异源表达等合 成生物学技术, 从这些链需菌菌株中发现更多活性优 异、结构新颖的天然产物值得期待[117,134-136].

\section{References}

[1] Castro, J. F.; Razmilic, V.; Gomez-Escribano, J. P.; Andrews, B.; Asenjo, J.; Bibb, M. Antonie Van Leeuwenhoek 2018, 111, 1433.

[2] Procopio, R. E.; Silva, I. R.; Martins, M. K.; Azevedo, J. L.; Araujo, J. M. Braz. J. Infect. Dis. 2012, 16, 466 .

[3] Berdy, J. J. Antibiot. (Tokyo) 2005, 58, 1.

[4] Chen, S.; Kinney, W. A.; Van Lanen, S. World J. Microbiol. Biotechnol. 2017, 33 .

[5] Newman, D. J.; Cragg, G. M. J. Nat. Prod. 2020, 83, 770

[6] Mogul, R.; Vaishampayan, P.; Bashir, M.; McKay, C. P.; Schubert, K.; Bornaccorsi, R.; Gomez, E.; Tharayil, S.; Payton, G.; Capra, J.; Andaya, J.; Bacon, L.; Bargoma, E.; Black, D.; Boos, K.; Brant, M.; Chabot, M.; Chau, D.; Cisneros, J.; Chu, G.; Curnutt, J.; DiMizio, J.; Engelbrecht, C.; Gott, C.; Harnoto, R.; Hovanesian, R.; Johnson, S.; Lavergne, B.; Martinez, G.; Mans, P.; Morales, E.; Oei, A.; Peplow, G.; Piaget, R.; Ponce, N.; Renteria, E.; Rodriguez, V.; Rodriguez, J.; Santander, M.; Sarmiento, K.; Scheppelmann, A.; Schroter, G.; Sexton, D.; Stephenson, J.; Symer, K.; Russo-Tait, T.; Weigel, B.; Wilhelm, M. B. Front. Microbiol. 2017, 8, 1974.

[7] Lewis, K. Cell 2020, 181, 29.

[8] Berdy, J. J. Antibiot. (Tokyo) 2012, 65, 385.

[9] Hutchings, M. I.; Truman, A. W.; Wilkinson, B. Curr. Opin. Microbiol. 2019, 51,72 .

[10] Sayed, A. M.; Hassan, M. H. A.; Alhadrami, H. A.; Hassan, H. M.; Goodfellow, M.; Rateb, M. E. J. Appl. Microbiol. 2020, 128, 630.

[11] Kamjam, M.; Sivalingam, P.; Deng, Z.; Hong, K. Front. Microbiol. 2017,8 .

[12] Yang, Z.; He, J.; Wei, X.; Ju, J.; Ma, J. Appl. Microbiol. Biotechnol. 2020, $104,67$.

[13] Zhao, Y.-C.; Zhu, T.-H.; Zhu, W.-M.; Chin. J. Org. Chem. 2013, 33, 1195 (in Chinese). (赵成英, 朱统汉, 朱伟明, 有机化学, 2013, 33, 1195.)

[14] Rateb, M. E.; Ebel, R.; Jaspars, M. Antonie Van Leeuwenhoek 2018, $111,1467$.

[15] Sivalingam, P.; Hong, K.; Pote, J.; Prabakar, K. Int. J. Microbiol. 2019, 5283948 .

[16] Skropeta, D. Nat. Prod. Rep. 2008, 25, 1131.

[17] Access on December 7, MarinLit Database, RSC. Available online: http://pubs.rsc.org/marinlit/
[18] Schupp, P. J.; Kohlert-Schupp, C.; Whitefield, S.; Engemann, A.; Rohde, S.; Hemscheidt, T.; Pezzuto, J. M.; Kondratyuk, T. P.; Park, E.-J.; Marler, L.; Rostama, B.; Wright, A. D. Nat. Prod. Commun. 2009, 4, 1717.

[19] Skropeta, D.; Wei, L. Nat. Prod. Rep. 2014, 31, 999.

[20] Weiss, C.; Figueras, E.; Borbely, A. N.; Sewald, N. J. Pept. Sci. 2017, 23, 514

[21] Carroll, A. R.; Copp, B. R.; Davis, R. A.; Keyzers, R. A.; Prinsep, M. R. Nat. Prod. Rep. 2020, 37, 175.

[22] Song, Y.; Yang, J.; Yu, J.; Li, J.; Yuan, J.; Wong, N.-K.; Ju, J. J. Antibiot. 2020, 73, 542 .

[23] Hohmann, C.; Schneider, K.; Bruntner, C.; Irran, E.; Nicholson, G.; Bull, A. T.; Jones, A. L.; Brown, R.; Stach, J. E.; Goodfellow, M.; Beil, W.; Kramer, M.; Imhoff, J. F.; Sussmuth, R. D.; Fiedler, H. P. J. Antibiot. (Tokyo) 2009, 62, 99.

[24] Nachtigall, J.; Schneider, K.; Bruntner, C.; Bull, A. T.; Goodfellow, M.; Zinecker, H.; Imhoff, J. F.; Nicholson, G.; Irran, E.; Sussmuth, R. D.; Fiedler, H. P. J. Antibiot. (Tokyo) 2011, 64, 453.

[25] Ueki, M.; Ueno, K.; Miyadoh, S.; Abe, K.; Shibata, K.; Taniguchi, M.; Oi, S. J. Antibiot. (Tokyo) 1993, 46, 1089.

[26] Sao, S.; Kajiura, T.; Noguchi, M.; Takehana, K.; Kobayashi, T.; Tsuji, T. J. Antibiot. 2001, 54, 102.

[27] Sommer, P. S.; Almeida, R. C.; Schneider, K.; Beil, W.; Sussmuth, R. D.; Fiedler, H. P. J. Antibiot. (Tokyo) 2008, 61, 683.

[28] Losada, A. A.; Cano-Prieto, C.; Garcia-Salcedo, R.; Brana, A. F.; Mendez, C.; Salas, J. A.; Olano, C. Microb. Biotechnol. 2017, 10, 873.

[29] Hughes, C. C.; MacMillan, J. B.; Gaudencio, S. P.; Jensen, P. R.; Fenical, W. Angew. Chem. Int. Ed. 2009, 48, 725.

[30] Wei, R.-B. X., T.; Li, J.; Jiang, Z.-D.; Paul, R. J.; William, F. Bioorg. Med. Chem. Lett. 1999, 9, 2003.

[31] Wei, R. B.; Xi, T.; Li, J.; Wang, P.; Li, F. C.; Lin, Y. C.; Qin, S. Mar. Drugs 2011, 9, 359 .

[32] Chen, C.; Wang, J.; Guo, H.; Hou, W.; Yang, N.; Ren, B.; Liu, M.; Dai, H.; Liu, X.; Song, F.; Zhang, L. Appl. Microbiol. Biotechnol. 2013, 97, 3885.

[33] Vieweg, L.; Reichau, S.; Schobert, R.; Leadlay, P. F.; Sussmuth, R. D. Nat. Prod. Rep. 2014, 31, 1554

[34] Pan, H. Q.; Zhang, S. Y.; Wang, N.; Li, Z. L.; Hua, H. M.; Hu, J. C.; Wang, S. J. Mar. Drugs 2013, 11, 3891.

[35] Song, C.-F. P, H.-Q.; Hu, J.-C. Chin. J. Antibiot. 2015, 40, 721 (in Chinese). (宋春风，潘华奇，胡江春，中国抗生素杂志，2015，40, 721.)

[36] Brana, A. F.; Sarmiento-Vizcaino, A.; Osset, M.; Perez-Victoria, I.; Martin, J.; de Pedro, N.; de la Cruz, M.; Diaz, C.; Vicente, F.; Reyes, F.; Garcia, L. A.; Blanco, G. Mar. Drugs 2017, 15, 144.

[37] Luo, M.; Tang, L.; Dong, Y.; Huang, H.; Deng, Z.; Sun, Y. Nat. Prod. Res. 2020, 27, 1.

[38] Prudhomme, M. Eur. J. Med. Chem. 2003, 38, 123.

[39] Du, Y. L.; Ryan, K. S. Curr. Opin. Chem. Biol. 2016, 31, 74

[40] Bharate, S. B.; Sawant, S. D.; Singh, P. P.; Vishwakarma, R. A. Chem. Rev. 2013, 113, 6761.

[41] Dowlati, A.; Posey, J.; Ramanathan, R. K.; Rath, L.; Fu, P.; Chak, A.; Krishnamurthi, S.; Brell, J.; Ingalls, S.; Hoppel, C. L.; Ivy, P.; Remick, S. C. Cancer Chemother. Pharmacol. 2009, 65, 73 .

[42] Fischer, T.; Stone, R. M.; Deangelo, D. J.; Galinsky, I.; Estey, E.; Lanza, C.; Fox, E.; Ehninger, G.; Feldman, E. J.; Schiller, G. J.; Klimek, V. M.; Nimer, S. D.; Gilliland, D. G.; Dutreix, C.; Huntsman-Labed, A.; Virkus, J.; Giles, F. J. J. Clin. Oncol. 2010, 28, 4339.

[43] Stone, R. M.; Mandrekar, S.; Sanford, B. L.; Geyer, S.; Bloomfield, C. D.; Dohner, K.; Thiede, C.; Marcucci, G.; Lo-Coco, F.; Klisovic, R. B.; Wei, A.; Sierra, J.; Sanz, M. A.; Brandwein, J. M.; de Witte, T.; Niederwieser, D.; Appelbaum, F. R.; Medeiros, B. C.; Tallman, M. S.; Krauter, J.; Schlenk, R. F.; Ganser, A.; Serve, H.; Ehninger, G.; Amadori, S.; Larson, R. A.; Dohner, H. Blood 2015, 126.

[44] Zhang, W.; Liu, Z.; Li, S.; Yang, T.; Zhang, Q.; Ma, L.; Tian, X.; Zhang, H.; Huang, C.; Zhang, S.; Ju, J.; Shen, Y.; Zhang, C. Org. 
Lett. 2012, 14, 3364.

[45] Liu, Z.; Ma, L.; Zhang, L.; Zhang, W.; Zhu, Y.; Chen, Y.; Zhang, W.; Zhang, C. Org. Biomol. Chem. 2019, 17, 1053.

[46] Zhang, W.; Ma, L.; Li, S.; Liu, Z.; Chen, Y.; Zhang, H.; Zhang, G.; Zhang, Q.; Tian, X.; Yuan, C.; Zhang, S.; Zhang, W.; Zhang, C. J. Nat. Prod. 2014, 77, 1887.

[47] Carro, L.; Golinska, P.; Nouioui, I.; Bull, A. T.; Igual, J. M.; Andrews, B. A.; Klenk, H.-P.; Goodfellow, M. Int. J. Syst. Evol. Microbiol. 2019, 69, 3426.

[48] Busarakam, K.; Brown, R.; Bull, A. T.; Tan, G. Y.; Zucchi, T. D.; da Silva, L. J.; de Souza, W. R.; Goodfellow, M. Antonie Van Leeuwenhoek 2016, 109, 319.

[49] Zhang, W.; Li, S.; Zhu, Y.; Chen, Y.; Chen, Y.; Zhang, H.; Zhang, G.; Tian, X.; Pan, Y.; Zhang, S.; Zhang, W.; Zhang, C. J. Nat. Prod. 2014, 77, 388

[50] Huang, H.; Yang, T.; Ren, X.; Liu, J.; Song, Y.; Sun, A.; Ma, J.; Wang, B.; Zhang, Y.; Huang, C.; Zhang, C.; Ju, J. J. Nat. Prod. 2012, 75, 202.

[51] Zhu, X.; Duan, Y.; Cui, Z.; Wang, Z.; Li, Z.; Zhang, Y.; Ju, J.; Huang, H. J. Antibiot. (Tokyo) 2017, 70, 819.

[52] Lai, Z.; Yu, J.; Ling, H.; Song, Y.; Yuan, J.; Ju, J.; Tao, Y.; Huang, H. Planta Med. 2018, 84, 201.

[53] Yao, Y.; Sun, S.; Cao, M.; Mao, M.; He, J.; Gai, Q.; Qin, Y.; Yao, X.; Lu, H.; Chen, F.; Wang, W.; Luo, M.; Zhang, H.; Huang, H.; Ju, J.; Bian, X. W.; Wang, Y. ACS Chem. Neurosci. 2020, 11, 2256.

[54] Pan, H.-Q.; Zhang, S.-Y.; Wang, N.; Li, Z.-L.; Hua, H.-M.; Hu, J.-C.; Wang, S.-J. Mar. Drugs 2013, 11, 3891.

[55] Um, S.; Kim, Y. J.; Kwon, H.; Wen, H.; Kim, S. H.; Kwon, H. C.; Park, S.; Shin, J.; Oh, D. C. J. Nat. Prod. 2013, 76, 873.

[56] Yang, X. W.; Peng, K.; Liu, Z.; Zhang, G. Y.; Li, J.; Wang, N.; Steinmetz, A.; Liu, Y. J. Nat. Prod. 2013, 76, 2360.

[57] Liu, N.; Shang, F.; Xi, L.; Huang, Y. Mar. Drugs 2013, 11, 1524.

[58] Liu, N.; Song, F.; Shang, F.; Huang, Y. Mar. Drugs 2015, 13, 6247.

[59] Pesic, A.; Baumann, H. I.; Kleinschmidt, K.; Ensle, P.; Wiese, J.; Sussmuth, R. D.; Imhoff, J. F. Mar. Drugs 2013, 11, 4834.

[60] You, Z. Y.; Wang, Y. H.; Zhang, Z. G.; Xu, M. J.; Xie, S. J.; Han, T. S.; Feng, L.; Li, X. G.; Xu, J. Mar. Drugs 2013, 11, 4035.

[61] Sun, C.; Yang, Z.; Zhang, C.; Liu, Z.; He, J.; Liu, Q.; Zhang, T.; Ju, J.; Ma, J. Org. Lett. 2019, 21, 1453.

[62] Wang, C.; Xu, Y.-J.; Huang, X.-L.; Hao, J.-J.; Zhu, W.-M.; Chin. Marine Drugs 2016, 35, 1 (in Chinese).

(王祄, 徐亚娟, 黄小龙, 郝杰杰, 朱伟明, 中国海洋药物, 2016, $35,1$.

[63] Wang, C.; Wang, L.; Fan, J.; Sun, K.; Zhu, W. Chin. J. Org. Chem. 2017, 37, 658 (in Chinese).

(王聪, 王立平, 范杰, 孙坤来, 朱伟明, 有机化学, 2017, 37, 658.)

[64] Song, Y.; Li, Q.; Liu, X.; Chen, Y.; Zhang, Y.; Sun, A.; Zhang, W.; Zhang, J.; Ju, J. J. Nat. Prod. 2014, 77, 1937.

[65] Pan, H. Q.; Yu, S. Y.; Song, C. F.; Wang, N.; Hua, H. M.; Hu, J. C.; Wang, S. J. J. Microbiol. Biotechnol. 2015, 25, 353.

[66] Zhou, X.; Huang, H. B.; Li, J.; Song, Y. X.; Jiang, R. W.; Liu, J.; Zhang, S.; Hua, Y.; Ju, J. H. Tetrahedron 2014, 70, 7795.

[67] Liu, N.; Song, F.; Shang, F.; Huang, Y. Mar. Drugs 2015, 13, 6247.

[68] Ma, X.-Y. W., G.-W.; Zhang, Z.-Y.; Geng, T.-T.; Sun, X.-X.; Yang, D.-H.; Tang, X.-X.; Ma, M. J. Chin. Chem. Pharm. Soc. 2019, 28 , 835 (in Chinese). (马学洋, 王贵阳, 张中义, 耿彤粀, 孙晓旭, 杨东辉, 汤熙祥, 马明, 药物科学杂志, 2019, 28, 835.)

[69] Bao, J.; He, F.; Li, Y.; Fang, L.; Wang, K.; Song, J.; Zhou, J.; Li, Q.; Zhang, H. J. Antibiot. (Tokyo) 2018, 71, 1018.

[70] Schmitz, J.; Gilberg, E.; Loser, R.; Bajorath, J.; Bartz, U.; Gutschow, M. Bioorg. Med. Chem. 2019, 27, 1.

[71] Takehana, Y.; Umekita, M.; Hatano, M.; Kato, C.; Sawa, R.; Igarashi, M. J. Antibiot. (Tokyo) 2017, 70, 611.

[72] Ortiz-Lopez, F. J.; Alcalde, E.; Sarmiento-Vizcaino, A.; Diaz, C.; Cautain, B.; Garcia, L. A.; Blanco, G.; Reyes, F. Mar. Drugs 2018, 16,371 .
[73] Jang, K. H.; Nam, S. J.; Locke, J. B.; Kauffman, C. A.; Beatty, D. S.; Paul, L. A.; Fenical, W. Angew. Chem. Int. Ed. 2013, 52, 7822.

[74] Rodriguez, V.; Martin, J.; Sarmiento-Vizcaino, A.; de la Cruz, M.; Garcia, L. A.; Blanco, G.; Reyes, F. Mar. Drugs 2018, 16, 406.

[75] Shen, X.; Wang, X.; Huang, T.; Deng, Z.; Lin, S. Biomolecules 2020, 10, 684 .

[76] Niu, S.; Li, S.; Chen, Y.; Tian, X.; Zhang, H.; Zhang, G.; Zhang, W.; Yang, X.; Zhang, S.; Ju, J.; Zhang, C. J. Antibiot. (Tokyo) 2011, 64, 711 .

[77] Luo, M.; Tang, G.; Ju, J.; Lu, L.; Huang, H. Nat. Prod. Res. 2016, 30,138

[78] Wang, T.; Jiang, Y.; Ma, K. X.; Li, Y. Q.; Huang, R.; Xie, X. S.; Wu, S. H. Chem. Biodiversity 2014, 11, 929.

[79] Rateb, M. E.; Houssen, W. E.; Harrison, W. T.; Deng, H.; Okoro, C. K.; Asenjo, J. A.; Andrews, B. A.; Bull, A. T.; Goodfellow, M.; Ebel, R.; Jaspars, M. J. Nat. Prod. 2011, 74, 1965

[80] Cha, J. W.; Lee, S. I.; Kim, M. C.; Thida, M.; Lee, J. W.; Park, J. S.; Kwon, H. C. Bioorg. Med. Chem. Lett. 2015, 25, 5083.

[81] Chen, Y.; Neilson, J. W.; Kushwaha, P.; Maier, R. M.; Barberán, A. ISME J. 2020.

[82] Wyman, S. K.; Avila-Herrera, A.; Nayfach, S.; Pollard, K. S. PLoS One 2018, 13, e0205749.

[83] Galand, P. E.; Pereira, O.; Hochart, C.; Auguet, J. C.; Debroas, D. ISME J. 2018, 12, 2470.

[84] Velez, P.; Espinosa-Asuar, L.; Figueroa, M.; Gasca-Pineda, J.; Aguirre-von-Wobeser, E.; Eguiarte, L. E.; Hernandez-Monroy, A.; Souza, V. Front. Microbiol. 2018, 9, 1755.

[85] Ziemert, N.; Alanjary, M.; Weber, T. Nat. Prod. Rep. 2016, 33, 988.

[86] Gomez-Escribano, J. P.; Castro, J. F.; Razmilic, V.; Chandra, G.; Andrews, B.; Asenjo, J. A.; Bibb, M. J. BMC Genomics 2015, 16.

[87] Wakefield, J.; Hassan, H. M.; Jaspars, M.; Ebel, R.; Rateb, M. E. Front. Microbiol. 2017, 8.

[88] Baltz, R. H. J. Ind. Microbiol. Biotechnol. 2017, 44, 573.

[89] Schulz, D.; Beese, P.; Ohlendorf, B.; Erhard, A.; Zinecker, H.; Dorador, C.; Imhoff, J. F. J. Antibiot. (Tokyo) 2011, 64, 763.

[90] Rateb, M. E.; Houssen, W. E.; Arnold, M.; Abdelrahman, M. H.; Deng, H.; Harrison, W. T.; Okoro, C. K.; Asenjo, J. A.; Andrews, B A.; Ferguson, G.; Bull, A. T.; Goodfellow, M.; Ebel, R.; Jaspars, M. J. Nat. Prod. 2011, 74, 1491 .

[91] Nachtigall, J.; Kulik, A.; Helaly, S.; Bull, A. T.; Goodfellow, M.; Asenjo, J. A.; Maier, A.; Wiese, J.; Imhoff, J. F.; Sussmuth, R. D.; Fiedler, H. P. J. Antibiot. (Tokyo) 2011, 64, 775.

[92] Santhanam, R.; Okoro, C. K.; Rong, X.; Huang, Y.; Bull, A. T.; Weon, H. Y.; Andrews, B. A.; Asenjo, J. A.; Goodfellow, M. Int. J. Syst. Evol. Microbiol. 2012, 62, 2680.

[93] Kurapova, A. I.; Zenova, G. M.; Sudnitsyn, I. I.; Kizilova, A. K.; Manucharova, N. A.; Norovsuren, Z.; Zvyagintsev, D. G. Microbiology 2012, 81, 98 .

[94] Meklat, A.; Sabaou, N.; Zitouni, A.; Mathieu, F.; Lebrihi, A. Appl. Environ. Microbiol. 2011, 77, 6710.

[95] Ibeyaima, A.; Singh, A. K.; Lal, R.; Gupta, S.; Goodfellow, M.; Sarethy, I. P. Antonie Van Leeuwenhoek 2018, 111, 2141.

[96] Abdelkader, M. S. A.; Philippon, T.; Asenjo, J. A.; Bull, A. T.; Goodfellow, M.; Ebel, R.; Jaspars, M.; Rateb, M. E. J. Antibiot. (Tokyo) 2018, 71, 425 .

[97] Bull, A. T.; Asenjo, J. A. Antonie Van Leeuwenhoek 2013, 103, 1173.

[98] Cortes-Abayay, C.; Dorador, C.; Schumann, P.; Andrews, B.; Asenjo, J.; Nouioui, I. Int. J. Syst. Evol. Microbiol. 2019, 69, 2315.

[99] Okoro, C. K.; Brown, R.; Jones, A. L.; Andrews, B. A.; Asenjo, J. A.; Goodfellow, M.; Bull, A. T. Antonie Van Leeuwenhoek 2009, 95, 121.

[100] Elsayed, S. S.; Trusch, F.; Deng, H.; Raab, A.; Prokes, I.; Busarakam, K.; Asenjo, J. A.; Andrews, B. A.; van West, P.; Bull, A. T.; Goodfellow, M.; Yi, Y.; Ebel, R.; Jaspars, M.; Rateb, M. E. J. Org. Chem. 2015, 80, 10252.

[101] Gomez-Escribano, J. P.; Castro, J. F.; Razmilic, V.; Jarmusch, S. A.; Saalbach, G.; Ebel, R.; Jaspars, M.; Andrews, B.; Asenjo, J. A.; Bibb, M. J. Appl. Environ. Microbiol. 2019, 85. 
[102] Cortes-Albayay, C.; Jarmusch, S. A.; Trusch, F.; Ebel, R.; Andrews, B. A.; Jaspars, M.; Asenjo, J. A. J. Org. Chem. 2020, 85, 1661.

[103] Yekkour, A.; Meklat, A.; Bijani, C.; Toumatia, O.; Errakhi, R.; Lebrihi, A.; Mathieu, F.; Zitouni, A.; Sabaou, N. Lett. Appl. Microbiol. 2015, 60, 589.

[104] Nithya, K.; Muthukumar, C.; Biswas, B.; Alharbi, N. S.; Kadaikunnan, S.; Khaled, J. M.; Dhanasekaran, D. Microbiol. Res. 2018, $207,116$.

[105] Srinivas, T. N.; Anil Kumar, P.; Tank, M.; Sunil, B.; Poorna, M.; Zareena, B.; Shivaji, S. Int. J. Syst. Evol. Microbiol. 2015, 65, 2391.

[106] Zhang, S.; Gui, C.; Shao, M.; Kumar, P. S.; Huang, H.; Ju, J. Nat. Prod. Res. 2018, 34, 1499.

[107] Meena, B.; Anburajan, L.; Vinithkumar, N. V.; Kirubagaran, R.; Dharani, G. Microb. Pathog. 2019, 132, 129.

[108] Sottorff, I.; Wiese, J.; Imhoff, J. F. Int. Microbiol. 2019, 22, 377.

[109] Kelly, L. C.; Cockell, C. S.; Thorsteinsson, T.; Marteinsson, V.; Stevenson, J. Microb. Ecol. 2014, 68, 504.

[110] Santhanam, R.; Rong, X.; Huang, Y.; Andrews, B. A.; Asenjo, J. A.; Goodfellow, M. Antonie Van Leeuwenhoek 2013, 103, 367.

[111] Kay, S.; Pathom-aree, W.; Cheeptham, N. Chiang. Mai J. Sci. 2013, 40, 26.

[112] Um, S.; Choi, T. J.; Kim, H.; Kim, B. Y.; Kim, S. H.; Lee, S. K.; Oh, K. B.; Shin, J.; Oh, D. C. J. Org. Chem. 2013, 78, 12321

[113] Hur, J.; Jang, J.; Sim, J.; Son, W. S.; Ahn, H. C.; Kim, T. S.; Shin, Y. H.; Lim, C.; Lee, S.; An, H.; Kim, S. H.; Oh, D. C.; Jo, E. K.; Jang, J.; Lee, J.; Suh, Y. G. Angew. Chem. Int. Ed. 2018, 57, 3069.

[114] Kim, T. S.; Shin, Y. H.; Lee, H. M.; Kim, J. K.; Choe, J. H.; Jang, J. C.; Um, S.; Jin, H. S.; Komatsu, M.; Cha, G. H.; Chae, H. J.; Oh, D. C.; Jo, E. K. Sci. Rep. 2017, 7, 3431.

[115] Wakefield, J.; Hassan, H. M.; Jaspars, M.; Ebel, R.; Rateb, M. E. Front Microbiol 2017, 8, 1284.

[116] Bae, M.; An, J. S.; Bae, E. S.; Oh, J.; Park, S. H.; Lim, Y.; Ban, Y. H.; Kwon, Y.; Cho, J. C.; Yoon, Y. J.; Lee, S. K.; Shin, J.; Oh, D. C. Org. Lett. 2019, 21, 3635.

[117] Bae, M.; An, J. S.; Hong, S.-H.; Bae, E. S.; Chung, B.; Kwon, Y.; Hong, S.; Oh, K.-B.; Shin, J.; Lee, S. K.; Oh, D.-C. Mar. Drugs 2020,18 .

[118] Son, S.; Jang, M.; Lee, B.; Hong, Y. S.; Ko, S. K.; Jang, J. H.; Ahn, J. S. J. Nat. Prod. 2018, 81, 2205.

[119] Zeng, Y.-X.; Chen, B.; Zou, Y.; Zheng, T.-L. J. Microbiol. 2008, 48, 695 (in Chinese).

(曾)胤新, 陈波, 邹扬, 郑天凌, 微生物学报, 2008, 48, 695.)
[120] Tripathi, V. C.; Satish, S.; Horam, S.; Raj, S.; lal, A.; Arockiaraj, J.; Pasupuleti, M.; Dikshit, D. K. Polar Sci. 2018, 18, 147.

[121] Tian, Y.; Li, Y. L.; Zhao, F. C. Mar. Drugs 2017, 15.

[122] Silva, L. J.; Crevelin, E. J.; Souza, D. T.; Lacerda-Junior, G. V.; de Oliveira, V. M.; Ruiz, A.; Rosa, L. H.; Moraes, L. A. B.; Melo, I. S. Sci. Rep. 2020, 10, 13870.

[123] Yang, A.-G.; Si, L.-L.; Shi, Z.-P.; Tian, L.; Liu, D.; Zhou, D.-M. Proksch, P.; Lin, W.-H. Org. Lett. 2013, 15, 5366.

[124] Su, S. S.; Tian, L.; Chen, G.; Li, Z. Q.; Xu, W. F.; Pei, Y. H. J. Asian Nat. Prod. Res. 2013, 15, 265.

[125] Philkhana, S. C.; Jachak, G. R.; Gunjal, V. B.; Dhage, N. M.; Bansode, A. H.; Reddy, D. S. Tetrahedron Lett. 2015, 56, 1252.

[126] Moon, K.; Ahn, C. H.; Shin, Y.; Won, T. H.; Ko, K.; Lee, S. K.; Oh, K. B.; Shin, J.; Nam, S. I.; Oh, D. C. Mar. Drugs 2014, 12, 2526.

[127] Mei, D.-H. Master's Thesis, Shanghai Ocean University, Shanghai, 2015 (in Chinese). (梅东海，硕士论文，上海海洋大学，上海, 2015.)

[128] Jiang, S.-P. Master's Thesis, Shanghai Ocean University, Shanghai 2015 (in Chinese). (江昇平，硕士论文，上海海洋大学，上海, 2015.)

[129] Kim, D.; Lee, E. J.; Lee, J.; Leutou, A. S.; Shin, Y. H.; Choi, B.; Hwang, J. S.; Hahn, D.; Choi, H. Chin, J.; Cho, S. J.; Hong, Y. D.; Ko, J.; Seong, C. N.; Maloney, K. N.; Oh, D. C.; Yang, I.; Hwang, H.; Nam, S. J. Mar. Drugs 2018, 16, 130.

[130] Shen, J.; Fan, Y.; Zhu, G.; Chen, H.; Zhu, W.; Fu, P. Org. Lett. 2019, 21, 4816.

[131] Shen, J. J.; Wang, J.; Chen, H.; Wang, Y.; Zhu, W. M.; Fu, P. Org. Chem. Front. 2020, 7, 310.

[132] Liu, D.; Yang, A.; Wu, C.; Guo, P.; Proksch, P.; Lin, W. Bioorg. Med. Chem. Lett. 2014, 24, 5288.

[133] Kim, D.; Lee, E. J.; Lee, J.; Leutou, A. S.; Shin, Y. H.; Choi, B.; Hwang, J. S.; Hahn, D.; Choi, H.; Chin, J.; Cho, S. J.; Hong, Y. D.; Ko, J.; Seong, C. N.; Maloney, K. N.; Oh, D. C.; Yang, I.; Hwang, H.; Nam, S. J. Mar. Drugs 2018, 16.

[134] Tan, B.; Chen, S.; Zhang, Q.; Chen, Y.; Zhu, Y.; Khan, I.; Zhang, W.; Zhang, C. Org. Lett. 2020, 22, 1062.

[135] Liu, W.; Jannu, V. G.; Liu, Z.; Zhang, Q.; Jiang, X.; Ma, L.; Zhang, W.; Zhang, C.; Zhu, Y. Org. Biomol. Chem. 2020, 18, 3649.

[136] He, J.; Wei, X.; Yang, Z.; Li, Y.; Ju, J.; Ma, J. Mar. Drugs 2020, 18 216.

(Fan, Y.) 